JOURNAL OF

SYMPLECTIC GEOMETRY

Volume 2, Number 3, 279-308, 2004

\title{
SYMPLECTIC INVARIANTS OF SOME FAMILIES OF LAGRANGIAN $T^{3}$-FIBRATIONS
}

\author{
Ricardo Castaño Bernard
}

\begin{abstract}
We construct families of Lagrangian 3-torus fibrations resembling the topology of some of the singularities in Topological Mirror Symmetry [8]. We perform a detailed analysis of the affine structure on the base of these fibrations near their discriminant loci. This permits us to classify the aforementioned families up to fibre preserving symplectomorphism. The kind of degenerations we investigate give rise to a large number of symplectic invariants.
\end{abstract}

\section{Introduction}

There is increasing interest in the geometry of Calabi-Yau manifolds. Much of this interest is motivated by an intriguing relation between pairs of CalabiYau manifolds called Mirror Symmetry. This relation interchanges- in a highly non-trivial way -the complex structure of a Calabi-Yau manifold, $Y$, with the symplectic structure of its mirror, $\check{Y}$. There are several approaches to Mirror Symmetry; one of these is proposed by Strominger, Yau and Zaslow (SYZ) [21]. The SYZ Conjecture claims- based on string theoretic arguments - that the mirror relation can be explained in terms of certain duality between $T^{n}$-fibrations on a pair of mirror Calabi-Yau manifolds.

The purpose of this paper is primarily motivated by the SYZ Conjecture; we are interested in the symplectic geometry of Calabi-Yau manifolds fibred by tori. Let $(Y, J, \omega)$ be a compact Kähler manifold of $\operatorname{dim}_{\mathbb{C}}(Y)=n$ with complex structure $J$ and Kähler (symplectic) form $\omega$. We say that $Y$ is a Calabi-Yau manifold if the canonical line bundle has a non-vanishing global section $\Omega$ such that $c \Omega \wedge \bar{\Omega}=\omega^{n}$ for some constant $c[\mathbf{9}]$. A very popular example of a Calabi-Yau 3 -fold is the (smooth) quintic hypersurface in $\mathbb{P}^{4}$ defined by:

$$
x_{0} x_{1} x_{2} x_{3} x_{4}+t\left(x_{0}^{5}+x_{1}^{5}+x_{2}^{5}+x_{3}^{5}+x_{4}^{5}\right)=0,
$$


where $t \in D_{0} \subset \mathbb{C}$ a small punctured disk around 0 .

A submanifold $L$ of $Y$ is called Lagrangian if $\left.\omega\right|_{L}=0$ and $\operatorname{dim}_{\mathbb{R}} L=$ $n$. A Lagrangian submanifold $L$ satisfying $\left.\operatorname{Im} \Omega\right|_{L}=0$, is called special Lagrangian. This term was coined by Harvey and Lawson [13].

A first attempt to state the SYZ Conjecture using mathematical language can be outlined as follows (c.f. $[\mathbf{1 0}],[\mathbf{5}]$ and $[\mathbf{6}]$ ):

Conjecture 1.1. Let $Y$ and $\check{Y}$ be a mirror pair of Calabi-Yau $n$-folds satisfying certain additional conditions. Let $B$ be a compact connected manifold. Then there exists $C^{\infty}$ maps $f: Y \rightarrow B$ and $\check{f}: \check{Y} \rightarrow B$ such that for $b \in B$ the fibres $f^{-1}(b)$ and $\check{f}^{-1}(b)$ are special Lagrangian. There is a codimension 2 closed subset $\Delta \subseteq B$ such that the fibres $f^{-1}(b)$ and $\check{f}^{-1}(b)$ over $b \in B \backslash \Delta$ are dual $n$-tori.

The idea of duality in Conjecture 1.1 can be explained in the following way. First consider the $T^{n}$-bundle $f_{0}: Y_{0} \rightarrow B_{0}=B \backslash \Delta$, resulting from removing the singular fibres of $f$. Let $G$ be a group $(G=\mathbb{R}$ or $\mathbb{Z}$ for our purposes) and denote by $R^{k} f_{0 *}(G)$ the locally constant sheaf on $B_{0}$ induced by the presheaf $\mathcal{R}^{k} f_{0 *}(G)=\left\{U \mapsto H^{k}\left(f^{-1}(U), G\right), U \subseteq B\right\}$. Denote by $\mathcal{E}=R^{1} f_{0 *}(\mathbb{R}) \otimes C^{\infty}\left(B_{0}\right)$. This gives a rank $n$ vector bundle $\mathcal{E} \rightarrow B_{0}$ with $R^{1} f_{0 *}(\mathbb{Z})$ being a family of rank $n$ lattices lying inside $\mathcal{E}$. Letting $\check{Y}_{0}=\mathcal{E} /$ $R^{1} f_{0 *}(\mathbb{Z})$ we can define the dual of $f_{0}$ as the $T^{n}$-bundle:

$$
\check{f}_{0}: \check{Y}_{0} \rightarrow B_{0} \text {. }
$$

According to Conjecture 1.1 one expects to recover the mirror of $Y$ as a compactification of $\check{Y}_{0}$, obtained by means of gluing on suitable singular fibres. This method raises a number of issues demanding careful consideration. As pointed out in $[\mathbf{2 1}, \S 5]$, understanding the structure of the singular fibres is probably one of the crucial issues.

Conjecture 1.1 appears to be the right approach if one pays attention to the topology only, i.e., forgetting about the complex and symplectic structures and considers $Y$ and $\check{Y}$ as $C^{\infty}$ manifolds only. Under some mild assumptions on the singular fibres- they are assumed to be semi-stable, i.e., with unipotent monodromy -the SYZ duality explains a topological version of Mirror Symmetry for the quintic:

Theorem 1.2 (Gross [8]). Let $Q \subseteq \mathbb{P}^{4}$ be a smooth quintic 3-fold and $\Xi$ a 4-simplex. There is a $T^{3}$ fibration $g: Q \rightarrow \partial \Xi$ with semi-stable fibres. The dual $\check{g}: \check{Q} \rightarrow \partial \Xi$ has only semi-stable fibres and $\check{Q}$ is diffeomorphic to a mirror pair of $Q$.

Both fibrations $g$ and $\check{g}$ have the same discriminant locus which consists of a trivalent graph $\Gamma$ lying over the faces of $\partial \Xi$. There are three types of singular fibres present in both $Q$ and $\check{Q}$. Let $s \in \Gamma$ and let $Q_{s}$ be a singular 
fibre of either $g$ or $\check{g}$. Let $\left(b_{1}, b_{2}\right)$ where $b_{i}=\operatorname{rank} H^{i}\left(Q_{s}, \mathbb{Z}\right), i=1,2$. Then $Q_{s}$ can be one of the following types:

- type $(2,2)$. This fibre is $S^{1} \times I_{1}$, where $I_{1}$ is a Kodaira type $I_{1}$ fibre (a pinched torus). So, fibres of type $(2,2)$ are singular along a circle. Fibres of type $(2,2)$ lie over the edges of $\Gamma$;

- type $(1,2)$. This fibre is obtained by collapsing a torus $T^{2} \times\{p\}$ on $T^{2} \times S^{1}$ to a point. Fibres of this kind lie over some vertices of $\Gamma$;

- type $(2,1)$. Let $S \subset T^{2}$ be a "figure eight" (c.f. [8, fig. 2.2]). This fibre is obtained by collapsing the circles $\{p\} \times S^{1}, p \in S$, on $T^{2} \times S^{1}$ to a point. Fibres of this kind lie over some vertices of $\Gamma$.

The $(2,1)$ fibre is dual to the $(1,2)$ fibre (their local monodromy representations are dual), whereas the $(2,2)$ fibre is self-dual.

One can try to add on structures to the above topological picture. First, one can try to put suitable symplectic structures on $Q$ and $\check{Q}$ making $g$ and $\check{g}$ into Lagrangian fibrations. The next step would be to put suitable (almost) Calabi-Yau structures on $Q$ and $\check{Q}$ making $g$ and $\check{g}$ into special Lagrangian fibrations. Recent development on special Lagrangian geometry (c.f. Joyce [15]) suggests that this program may not be fully completed in the strong terms of Conjecture 1.1. This is not conclusive, however.

There has been some progress in the symplectic category. Wei-Dong Ruan [18], [20] constructs Lagrangian torus fibrations on the quintic. Ruan's method consists, roughly speaking, on a certain gradient flow deformation of a well known Lagrangian fibration on the normal crossing quintic to a neighbour non-singular quintic. This produces a piecewise $C^{\infty}$ fibration with codimension 1 discriminant locus. The topology of this fibration differs from the topological $T^{3}$ fibration in [8]. Ruan argues [19] that the codimension 1 discriminant can be deformed to codimension 2 , in which case the resulting fibration coincides, topologically, with the one in [8].

In this paper we are interested in the semi-global symplectic geometry in a neighbourhood of the singular fibres rather than in the global picture. We follow the spirit of $[\mathbf{8}]$ and construct singular local models of Lagrangian $T^{3}$ fibrations first. We are able to construct $C^{\infty}$ Lagrangian $T^{3}$ fibrations with singular fibres resembling the topology of the $(2,2)$ and $(1,2)$ fibres. To date there is no symplectic model for the $(2,1)$ fibre and it is not yet clear whether there exists a Lagrangian $T^{3}$ fibration on the quintic presenting singular fibres of type $(2,1)$.

In two dimensions, it is known that the Kodaira type $I_{1}$ degeneration of an elliptic fibration, has an infinite number of symplectic invariants (c.f. Vũ-Ngoc [22] and [1] for an alternative approach and for the $C^{k}$-symplectic case). We show that a similar behaviour appears in dimension $n \geq 3$ : the 
families of Lagrangian $T^{n}$ fibrations considered in this paper have infinite dimensional classifying spaces.

Gross and Wilson [11] and, independently, Kontsevich and Soibelman [16] propose an alternative interpretation of SYZ Conjecture, which can be regarded as a relaxed version of Conjecture 1.1. This new proposal posits that the SYZ Conjecture is true in certain limiting sense as the mirror pair of Calabi-Yau manifolds approach large complex structure limits. The SYZ duality is then interpreted as a certain kind of Legendre transform between (singular) affine structures on the bases of the fibrations. This conjecture is proved for K3 surfaces by Gross and Wilson [11]. For the case of the quintic, Gross constructs an affine structure on the complement of the discriminant locus (c.f. [9, §19.3]). This affine structure, in turn, induces a symplectic structure on the complement of the union of the singular fibres. The results here can be interpreted as a description of how this affine structure may become singular at the discriminant locus and the symplectic invariants arising from this degeneration.

Statement of the main results. Let $g: Q \rightarrow \partial \Xi$ be the $T^{3}$ fibration on the quintic as in Theorem 1.2. Let $s_{0} \in \Gamma$ and consider $U \subseteq \partial \Xi$ an open neighbourhood of $s_{0}$. Assume $U$ is small enough so that it contains at most one vertex of $\Gamma$ and such that $\Gamma \cap U$ is connected. Then $g^{-1}(U) \subseteq Q$ is a neighbourhood of the fibre $g^{-1}\left(s_{0}\right)$ and the restriction of $g$ to $g^{-1}(U)$ gives a $T^{3}$ fibration, $g^{-1}(U) \rightarrow U$, which is singular along $\Gamma \cap U$.

Now suppose there is a non-compact symplectic manifold $(X, \omega)$ together with a proper Lagrangian fibration $f: X \rightarrow B$. In addition, suppose there are diffeomorphisms $\Phi$ and $\phi$ giving a commutative diagram:

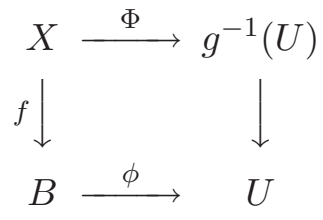

Let $\Delta=\phi^{-1}(\Gamma \cap U)$ and $b_{0}:=\phi^{-1}\left(s_{0}\right) \in \Delta$. Then $f$ is a Lagrangian $T^{3}$ fibration having $\Delta$ as discriminant locus and the fibre $X_{b_{0}}:=f^{-1}\left(b_{0}\right)$ is homeomorphic to the fibre $g^{-1}\left(s_{0}\right)$.

Definition 1.3. Let $\mathcal{L}\left(X_{b_{0}}\right)$ denote the set of triples $\mathcal{F}=(X, \omega, f)$ where $f$ : $(X, \omega) \rightarrow B$ is a Lagrangian fibration arising as in diagram (3). We say that two elements $(X, \omega, f)$ and $\left(X^{\prime}, \omega^{\prime}, f^{\prime}\right)$ in $\mathcal{L}\left(X_{b_{0}}\right)$ are symplectically equivalent if there is a symplectomorphism $\Psi: X \rightarrow X^{\prime}$ and a diffeomorphism $\psi: B \rightarrow$ $B$ such that $\psi\left(b_{0}\right)=b_{0}$ and $f^{\prime} \circ \Psi=\psi \circ f$. The set of equivalence classes under this relation will be denoted by $\widetilde{\mathcal{L}}\left(X_{b_{0}}\right)$. The elements of $\widetilde{\mathcal{L}}\left(X_{b_{0}}\right)$ can be regarded as germs of Lagrangian fibrations around the singular fibre $X_{b_{0}}$. 
There are three families to be considered: $\mathcal{L}(2,2), \mathcal{L}(1,2)$ and $\mathcal{L}(2,1)$ corresponding to $X_{b_{0}}$ of type $(2,2),(1,2)$ and $(2,1)$ respectively. Their discriminant loci are as depicted in Figure 1.

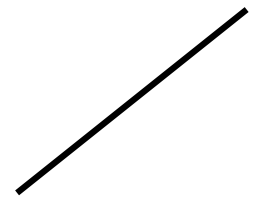

(a) $\kappa=2$

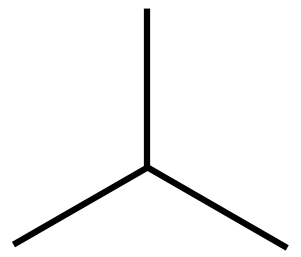

(b) $\kappa=1$

Figure 1. The discriminant loci $\Delta \subset B$.

Theorem 1.4. There is an element $\mathcal{F}_{H} \in \mathcal{L}(\kappa, 2), \kappa=1,2$, for each $H \in$ $C^{\infty}(B)$.

Theorem 1.5. The germs of fibrations of type $\widetilde{\mathcal{L}}(\kappa, 2), \kappa=1,2$, are classified by $C_{\Delta}^{\infty}(B)$, the space of germs of $C^{\infty}$ functions on $B$ vanishing at $\Delta$ to all orders.

\section{Preliminaries}

We recall the construction of action-angle variables on Lagrangian $T^{n}$ bundles. This is an extensively used technique in the context of Hamiltonian mechanics. The material presented here is standard (c.f. $[\mathbf{2}]$ and $[\mathbf{6}, \S 2]$ ).

Let $(X, \omega)$ be a symplectic manifold of dimension $2 n$ and $B$ a $n$-dimensional manifold. We shall assume $X$ and $B$ to be connected but not necessarily compact.

Definition 2.1. Let $f: X \rightarrow B$ be a proper $C^{\infty}$ map with connected fibres and denote by $\operatorname{Crit}(f) \subset X$ the set of points in $X$ where the differential $f_{*}$ is not surjective. Let $X^{\#}=X \backslash \operatorname{Crit}(f)$ and $f^{\#}$ denote the restriction of $f$ to $X^{\#}$. If the fibres of $f^{\#}$ are Lagrangian with respect to $\omega$ we say that $f$ is a Lagrangian fibration. We denote a Lagrangian fibration as a triple $\mathcal{F}=(X, \omega, f)$.

Observe that the Arnold-Liouville theorem implies that the regular fibres of $\mathcal{F}$ as in Definition 2.1 are diffeomorphic to $T^{n}$.

Definition 2.2. Let $\mathcal{F}=(X, \omega, f)$ be a Lagrangian fibration. Denote $X_{b}=$ $f^{-1}(b)$ and let $\operatorname{Crit}\left(X_{b}\right)=\operatorname{Crit}(f) \cap f^{-1}(b)$ the set of singular points of $X_{b}$. We say that $\mathcal{F}$ is admissible if

(1) Crit $\left(X_{b}\right)$ is connected and the fibres of $f^{\#}$ are connected; 
(2) $\Delta=f($ Crit $(f))$ is a closed codimension two subset of $B$ and

(3) $f^{\#}\left(X^{\#}\right)=B$ and for any point $x \in X^{\#}$ there is a local $C^{\infty}$ section of $f$ passing through $x$.

Observe that (3) in Definition 2.2 implies that $f$ does not have singular fibres dropping dimension. The fibres of $f^{\#}$ over $\Delta$ are diffeomorphic to $T^{k} \times \mathbb{R}^{n-k}$. From now on we only consider Lagrangian fibrations which are admissible.

Now let $B_{0}=B \backslash \Delta, X_{0}=f^{-1}\left(B_{0}\right)$ and $f_{0}=\left.f\right|_{X_{0}}$. The map, $f_{0}: X_{0} \rightarrow$ $B_{0}$ defines a $T^{n}$ fibre bundle, denoted by $\left(X_{0}, f_{0}\right)$. Consider $R^{n-1} f_{0 *} \mathbb{Z}$, a local system as defined in $\S 1$. Since $f_{0}$ is proper, one can identify the stalk $\left(R^{n-1} f_{0 *} \mathbb{Z}\right)_{b}$ with $H_{1}\left(X_{b}, \mathbb{Z}\right)$ using Poincaré duality.

Now consider $f^{\#}: X^{\#} \rightarrow B$ and let $X_{b}^{\#}=f^{\#-1}(b)$. We define a sheaf on $B$, with $\operatorname{stalk}^{1} H_{c}^{i}\left(X_{b}^{\#}, \mathbb{Z}\right)$ as follows. Let $U \subseteq B$ and consider the presheaf defined by $U \mapsto H_{c}^{i}\left(f^{\#-1}(U), \mathbb{Z}\right)$. The latter induces a sheaf, denoted $R_{c}^{i} f_{*}^{\#} \mathbb{Z}$, with stalk $\left(R_{c}^{i} f_{*}^{\#} \mathbb{Z}\right)_{b} \cong H_{c}^{i}\left(X_{b}^{\#}, \mathbb{Z}\right)$. Again, we can identify $H_{r}\left(X_{b}^{\#}, \mathbb{Z}\right)$ with $\left(R_{c}^{n-r} f_{*}^{\#} \mathbb{Z}\right)_{b}$.

Now we define a map $R_{c}^{n-1} f_{*}^{\#} \mathbb{Z} \hookrightarrow T_{B}^{*}$ as follows. For each $U \subseteq B$ open and $b \in U$ let $\gamma(b) \in H_{1}\left(X_{b}^{\#}, \mathbb{Z}\right) \cong H_{c}^{n-1}\left(X_{b}^{\#}, \mathbb{Z}\right), v \in T_{U, b}$ and $\tilde{v}$ a lifting of $v$. Define the map $(b, \gamma(b)) \mapsto \lambda_{b}$, where

$$
\lambda_{b}(v)=-\int_{\gamma(b)} \iota(\tilde{v}) \omega .
$$

This gives a local section $b \mapsto \lambda_{b}$ of $T_{B}^{*}$, i.e., a 1-form on $U \subseteq B$. One can check that the above formula does not depend on the lifting of $v$.

Definition 2.3. Let $\Lambda \subset T_{B}^{*}$ be the image of $R_{c}^{n-1} f_{*}^{\#} \mathbb{Z}$ under the map (4). We call $\Lambda$ the period lattice of $f$.

Now let us consider $R^{n-1} f_{0 *} \mathbb{Z}$. Choose a local section $\gamma$ of $R^{n-1} f_{0 *} \mathbb{Z}$ over an open set on $U \subseteq B_{0}$. The image of this section under the map (4) gives us a period 1-form $\lambda_{\gamma}$. This form is closed, since it is the differential of the action function:

$$
\mathcal{A}_{\gamma}(b)=\int_{\gamma(b)} \sigma .
$$

Here $\sigma$ is such that $d \sigma=\omega$. We can ensure that such a $\sigma$ always exists on $f_{0}^{-1}(U)$ by taking $U \subset B_{0}$ small enough. This means that the sections of $\Lambda$ are given locally by the image of a closed 1-form and, in particular, $\Lambda$ is Lagrangian with respect to the canonical symplectic structure on $T_{B}^{*}$.

\footnotetext{
${ }^{1}$ Here $H_{c}^{*}(\cdot, \mathbb{Z})$ denotes compactly supported cohomology with coefficients in $\mathbb{Z}$
} 
The above construction gives us an exact sequence:

$$
0 \longrightarrow R_{c}^{n-1} f_{*}^{\#} \mathbb{Z} \longrightarrow T_{B}^{*} \longrightarrow T_{B}^{*} / \Lambda \longrightarrow 0
$$

Proposition-Definition 2.4. The sequence (6) defines a symplectic manifold, $J^{\#}:=T_{B}^{*} / \Lambda$, and a Lagrangian fibration $\mathcal{J}_{f}: J^{\#} \rightarrow B$ with fibre $\mathcal{J}_{f}^{-1}(b)=T_{B, b}^{*} / \Lambda_{b}$. We call $\mathcal{J}_{f}$ the Jacobian fibration of $f$.

Proof. The Lagrangian nature of $\Lambda \subset T_{B}^{*}$ implies that translations over $\Lambda$ along the fibres of $T_{B}^{*}$ are symplectic transformations. Therefore, $J^{\#}:=T_{B}^{*} /$ $\Lambda$ inherits the canonical symplectic structure of $T_{B}^{*}$. The bundle projection $T_{B}^{*} \rightarrow B$ induces the map $\mathcal{J}_{f}: J^{\#} \rightarrow B$. It follows immediately that the fibres of $\partial_{f}$ are Lagrangian.

The following result is deduced from $[\mathbf{2}]$ (c.f. $[\mathbf{6}, \S 2])$ :

Theorem 2.5. Let $(X, \omega, f)$ be a proper Lagrangian fibration. Let $\mathcal{J}_{f}$ : $J^{\#} \rightarrow B$ be the Jacobian fibration of $f$ as defined in (2.4). Then,

(i) if $f^{\#}$ has a global section, $\Sigma: B \rightarrow X^{\#}$, then there is a fibre preserving diffeomorphism $\Psi: J^{\#} \rightarrow X^{\#}$;

(ii) if $\Sigma$ is Lagrangian, then the diffeomorphism in (i) is a symplectomorphism.

Duistermaat [2] observed that a Lagrangian $T^{n}$ bundle $f_{0}: X_{0} \rightarrow B_{0}$ has three invariants: its monodromy, its Chern class and $[\omega] \in H^{2}\left(X_{0}, \mathbb{R}\right)$. This tells us that, by taking $U \subset B_{0}$ contractible, we can define a set of actionangle (canonical) coordinates on $f^{-1}(U) \subset X$ which allows us to write $\omega$ on $f^{-1}(U)$ as the standard symplectic structure. Furthermore, the action coordinates provide $B_{0}$ with an integral affine structure.

\section{The family $\mathcal{L}(2,2)$}

Lagrangian $T^{2}$-fibrations with singular fibre of type $I_{1}$ are better known in symplectic geometry as focus-focus singularities; they appear in a number of "physically relevant" integrable Hamiltonian systems.

Theorem $3.1([\mathbf{1}])$. Let $D \subseteq \mathbb{C}$ be an open disk with coordinates $s=$ $s_{1}+\sqrt{-1} s_{2}$. For any function $h \in C^{\infty}(D)$ there is a Lagrangian $T^{2}$ fibration $\mathcal{F}=(\bar{X}, \omega, f)$ with singular fibre of focus-focus type and whose period lattice is generated by $\tau_{1}=-\log |s| d s_{1}+\operatorname{Arg}(s) d s_{2}+d h$ and $\tau_{2}=2 \pi d s_{2}$.

Remark 3.2. There is an alternative proof of Theorem 3.1 proposed by Vũ-Ngoc [22].

Proposition 3.3. Let $\bar{f}: \bar{X} \rightarrow D$ be a $T^{2}$ fibration as in Theorem 3.1 and $(0,1)$ an open interval. Let $X=\bar{X} \times S^{1} \times(0,1)$ and define $f: X \rightarrow D \times(0,1)$ to be the composition of the projection onto $\bar{X} \times(0,1)$ and $\bar{f} \times i d$. Then, 
there is a symplectic structure $\omega$ on $X$ making the fibres of $f$ Lagrangian. Furthermore the fibres over $\Delta=\{0\} \times(0,1)$ are diffeomorphic to $I_{1} \times S^{1}$.

Proof. Let $(r, \theta)$ be coordinates on $(0,1) \times S^{1}$. Define $\omega=\bar{\omega}+d r \wedge d \theta$. One can verify $f$ is Lagrangian with respect to $\omega$.

Definition 3.4. Let $M$ be a symplectic manifold and let $f_{1}, \ldots, f_{n} \in$ $C^{\infty}(M)$ define an integrable Hamiltonian system on $M$. Let $x \in M$ and let $\left(t_{j} ; x\right) \mapsto \phi_{j}^{t_{j}}(x)$ be the flow generated by the Hamiltonian vector field of $f_{j}$. We call $\left(t_{1}, \ldots, t_{n} ; x\right) \mapsto \phi_{n}^{t_{n}} \circ \cdots \circ \phi_{1}^{t_{1}}(x)$ the Poisson action of the system. If all flows $\phi_{j}$ are complete the Poisson action is an $\mathbb{R}^{n}$-action on $M$ which preserves the fibres of the map $x \mapsto\left(f_{1}(x), \ldots, f_{n}(x)\right)$.

Observe that for $f$ as in Proposition 3.3 all points $x \in \operatorname{Crit}(f)$ are nondegenerate and such that rank $\left.f_{*}\right|_{x}=1$. Regarding $f$ as an integrable system, one can check that the Poisson orbit of $x, \mathcal{O}_{x}$, is diffeomorphic to $S^{1}$ and each point in $\mathcal{O}_{x}$ is a rank one critical point. Rank one singular orbits of integrable systems are classified up to fibre preserving symplectomorphism. We state here a special case of a result due to Miranda and Tien-Zung [17].

Theorem $3.5([\mathbf{1 7}])$. Let $\left(M^{6}, \Omega, h\right)$ be a (not necessarily proper) Lagrangian fibration with a non-degenerate rank 1 singular orbit $\mathcal{O}$ of the Poisson action. Let $D^{4}$ be a 4-ball and let $V=D^{4} \times(-1,1) \times S^{1}$ be a symplectic 6 -manifold with canonical coordinates $\left(x_{j}, y_{j}, r, \theta\right)$. There exists a neighbourhood $U \subseteq M$ of $\mathcal{O}$ a Lagrangian fibration, $L: V \rightarrow D \times(-1,1)$, $L\left(x_{j}, y_{j}, r, \theta\right)=\left(q_{1}\left(x_{j}, y_{j}\right), q_{2}\left(x_{j}, y_{j}\right), r\right)$, and a fibre preserving symplectomorphism $\psi: U \rightarrow V$ sending $\mathcal{O}$ to $\left\{x_{i}=y_{i}=r=0\right\}$ and such that $q_{i}$ can be one of the following types:

$$
\begin{array}{ll}
\text { elliptic type: } & q_{i}=x_{i}^{2}+y_{i}^{2} \\
\text { hyperbolic type: } & q_{i}=x_{i} y_{i} \\
\text { focus-focus type: } & \left\{\begin{array}{l}
q_{i}=x_{i} y_{i}+x_{i+1} y_{i+1} \\
q_{i+1}=x_{i} y_{i+1}-x_{i+1} y_{i}
\end{array}\right.
\end{array}
$$

Definition 3.6. Let $\mathcal{F}=(X, \omega, f)$ be an admissible Lagrangian $T^{3}$ fibration. Let $x \in \operatorname{Crit}(X)$ be a non-degenerate rank 1 singular point and $\mathcal{O}_{x}$ its Poisson orbit. We say that $\mathcal{F}$ is a Lagrangian fibration of type $(2,2)$, denoted $\mathcal{F} \in$ $\mathcal{L}(2,2)$, if there is a neighbourhood $U \subset X$ of $\mathcal{O}_{x}$ such that $X=f^{-1}(f(U))$ and the following commutative diagram:

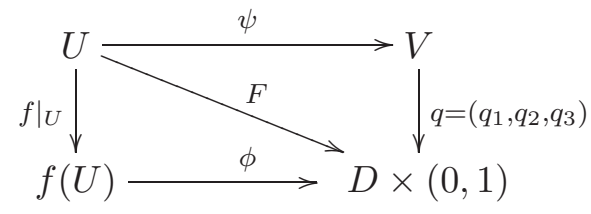


where $\psi: U \rightarrow V=D^{4} \times(0,1) \times S^{1}$ is a symplectomorphism, $\phi$ is a diffeomorphism and $q_{1}, q_{2}$ are of focus-focus type, $q_{3}=r$.

We shall denote $B=D \times(0,1)$ and $b=\left(b_{1}, b_{2}, b_{3}\right) \in B$. We can write $\phi=\left(\phi_{1}, \phi_{2}, \phi_{3}\right)$ and $\phi \circ f=\left(f_{1}, f_{2}, f_{3}\right)$, where $f_{i}=\phi_{i} \circ f$. If we think of $\psi$ as providing $U$ with canonical coordinates, then $\left.f_{j}\right|_{U}=q_{j}$ or, with slight abuse of notation, $\left.f\right|_{U}=F$ where $F\left(x_{i}, y_{i}\right)=\left(q_{1}, q_{2}, q_{3}\right)$ as in (7). We regard $F$ as the normal form for the family $\mathcal{L}(2,2)$.

Let $v_{q_{j}}$ be the Hamiltonian vector field corresponding to $q_{j}$ and let $g_{j}^{t}$ its flow. Let $\zeta_{1}=x_{1}+\sqrt{-1} x_{2}$ and $\zeta_{2}=y_{1}+\sqrt{-1} y_{2}$. Observe that $\bar{\zeta}_{1} \zeta_{2}=$ $q_{1}+\sqrt{-1} q_{2}$. The flows of $g_{j}: \mathbb{R} \times V \rightarrow V$ are given by:

$$
\begin{aligned}
& g_{1}^{t}\left(\zeta_{1}, \zeta_{2}, r, \theta\right)=\left(e^{t} \zeta_{1}, e^{-t} \zeta_{2}, r, \theta\right) \\
& g_{2}^{t}\left(\zeta_{1}, \zeta_{2}, r, \theta\right)=\left(e^{i t} \zeta_{1}, e^{i t} \zeta_{2}, r, \theta\right) \\
& g_{3}^{t}\left(\zeta_{1}, \zeta_{2}, r, \theta\right)=\left(\zeta_{1}, \zeta_{2}, r, \theta-t\right) .
\end{aligned}
$$

Observe that $g_{2}^{t}$ and $g_{3}^{t}$ generate a fibre-preserving $T^{2}$ action on $V$.

Lemma 3.7. Let $(X, \omega, f) \in \mathcal{L}(2,2)$. Then the compact fibres of $f^{\#}: X^{\#} \rightarrow$ $B$ are diffeomorphic to $T^{3}$ whereas the non-compact ones are diffeomorphic to $T^{2} \times \mathbb{R}$. There is an open neighbourhood $\mathcal{U} \subset X$ of Crit $(f)$ such that the fibres of $f_{\mathfrak{u}}:=\left.f\right|_{X \backslash u}$ are diffeomorphic to $T^{2} \times[0,1]$. Furthermore, $f_{\mathfrak{u}}$ defines a trivial fibre bundle.

Proof. The first part follows directly from the definition. For the second claim it is enough to take $\mathcal{U}$ a small connected neighbourhood of Crit $(f)$ which is invariant with respect to the $T^{2}$-action induced by $v_{q_{2}}$ and $v_{q_{3}}$ and redefine $B:=\phi \circ f(\mathcal{U})$. The triviality of $f_{\mathcal{U}}$ follows from the fact that $B$ is contractible.

Notice that $\left.f_{j}\right|_{u}=q_{j}$ implies that the vector fields $v_{q_{j}}$ extend vector Hamiltonian fields $v_{j}$ on $X$ which are tangent to the fibres, hence the flows $g_{i}^{t}$ extend to $X$. Since the fibres of $f$ are compact $g_{i}^{t}$ are complete.

Construction 3.8. Define an action $\Pi: \mathbb{R}^{3} \times X \rightarrow X,(T, x) \mapsto \Pi^{T}(x)$, $T=\left(t_{1}, t_{2}, t_{3}\right)$ as the composition of flows:

$$
\Pi(T, x):=g_{3}^{t_{3}} \circ g_{2}^{t_{2}} \circ g_{1}^{t_{1}}(x) .
$$

The restriction of $\Pi$ to $X^{\#}$ is just the Poisson action on $f^{\#}: X^{\#} \rightarrow B$, which is free and transitive along the fibres since $f^{\#-1}(b)$ is connected. This implies that for any two points $x, y \in f^{\#-1}(b)$, there is a multi-time $T=$ $T(x, y) \in \mathbb{R}^{3}$ such that $\Pi^{T}(x)=y$. Similarly, consider now the Hamiltonian vector fields $v_{q_{1}}, v_{q_{2}}, v_{q_{3}}$, on $\mathcal{U} \subseteq X$. In an analogous way, we can define an action on $\mathcal{U}, \Pi_{0}: R \times \mathcal{U} \rightarrow \mathcal{U}$, where $R \subseteq \mathbb{R}^{3}$ is some open set, as the composition of flows of $v_{q_{i}}$. Since $F^{-1}(b)$ is connected and non-singular for $b \in B_{0}=B \backslash \Delta, \Pi_{0}$ is transitive along the regular fibres of $F$. 
We are going to use the actions $\Pi$ and $\Pi_{0}$ to compute the period lattice of $(X, \omega, f)$. Let $\epsilon>0$ and write $s=b_{1}+\sqrt{-1} b_{2}, r=b_{3}$. Define, $\Sigma_{1}(s, r)=\left(\bar{s} / \epsilon, \epsilon, r, \theta_{0}\right)$ and $\Sigma_{2}(s, r)=\left(\epsilon, s / \epsilon, r, \theta_{0}\right), \theta_{0} \in S^{1}=\mathbb{R} / \mathbb{Z}$. These give sections of $F$ which lie inside $\mathcal{U}$ and do not intersect $\operatorname{Crit}(F)$. Now consider the equation:

$$
\Pi_{0}\left(T_{0}(b), \Sigma_{1}(b)\right)=\Sigma_{2}(b), b \in B_{0} .
$$

The solution $T_{0}=\left(\alpha_{1}, \alpha_{2}, \alpha_{3}\right)$ is determined by the system:

$$
\left\{\begin{array}{l}
e^{-\alpha_{1}+i \alpha_{2}} \cdot \epsilon=s / \epsilon \\
e^{\alpha_{1}+i \alpha_{2}} \cdot \bar{s} / \epsilon=\epsilon \\
\theta_{0}-\alpha_{3}=\theta_{0}
\end{array}\right.
$$

One verifies that the (primitive) solution to the system is

$$
\alpha_{1}=-\log |s|+2 \log \epsilon, \quad \alpha_{2}=\operatorname{Arg}(s), \quad \alpha_{3}=0 .
$$

Let $\mathcal{U}^{\prime}$ be a $T^{2}$ invariant neighbourhood of $\operatorname{Crit}(f), \mathcal{U}^{\prime} \subset \mathcal{U}$ as in Lemma 3.7. We can take $\mathcal{U}^{\prime}$ small enough so that we can regard $\Sigma_{1}$ and $\Sigma_{2}$ also as sections of the $T^{2} \times \mathbb{R}$ fibre bundle $f_{\mathfrak{U}^{\prime}}$ over $B$.

Proposition 3.9. Let $\Sigma_{1}$ and $\Sigma_{2}$ be sections of $f_{\mathfrak{U}^{\prime}}$ as above. The equation:

$$
\Pi^{T(b)}\left(\Sigma_{2}(b)\right)=\Sigma_{1}(b), \quad b \in B
$$

has a unique solution, $T(b)=\left(\eta_{1}(b), \eta_{2}(b), \eta_{3}(b)\right)$, which depends smoothly on $b \in B$.

Proof. A solution to equation (10) exists since the action $\Pi$ is transitive along the fibres of $f_{\mathcal{U}^{\prime}}$. We shall see that $T(b)$ depends smoothly on $b \in B$.

Let $S_{j}=\Sigma_{j}(B)$ and let $x_{0} \in S_{2}$ such that $f\left(x_{0}\right)=b_{0} \in B$. Then, there is $t_{0} \in \mathbb{R}^{3}$ such that $\Pi^{t_{0}}\left(x_{0}\right)=y_{0} \in S_{1}$. Let $U_{0}$ be a small neighbourhood of $x_{0}$ and let $R$ be a neighbourhood of $t_{0}$. Let $V_{0}$ be a neighbourhood of $y_{0}$ such that $f\left(V_{0}\right)=f\left(U_{0}\right) \subseteq B$. Define $P: R \times\left(U_{0} \cap S_{2}\right) \rightarrow V_{0}$, as $P(t, x)=\Pi^{t}(x)$. Notice that $S_{1}$ is transversal to the $\mathbb{R}^{3}$-orbit of $\Pi$ passing through a point $y \in S_{1}$. This implies that $P$ is transversal to $S_{1} \cap V_{0}$. Then, $\mathcal{P}:=P^{-1}\left(S_{1} \cap V_{0}\right)$ is a codimension 3 smooth submanifold of $R \times\left(U_{0} \cap S_{2}\right)$.

Now observe that since $\Pi$ is an action, the "time" derivative of $P$ evaluated at $\left(t_{0}, x_{0}\right)$ is non-singular. Then, $\mathcal{P}$ can be described locally as the graph of a $C^{\infty}$ map, $g: U_{0}^{\prime} \rightarrow R$, where $U_{0}^{\prime} \subseteq\left(S_{2} \cap U_{0}\right)$ is a small neighbourhood of $x_{0}$. Let $B^{\prime}=f\left(U_{0}^{\prime}\right)$ and define $T: B^{\prime} \subseteq B \rightarrow R$, as $T(b)=g \circ \Sigma_{2}(b)$ for $b \in B^{\prime}$. Then, $T$ is a $\mathbb{R}^{3}$-valued $C^{\infty}$ function such that $\Pi^{T(b)}\left(\Sigma_{2}(b)\right)=\Sigma_{1}(b)$. From Lemma 3.7 we know that $f_{\mathcal{U}^{\prime}}$ has trivial monodromy. Therefore these local solutions can be glued together to give a single-valued global solution, $T(b)=\left(\eta_{1}(b), \eta_{2}(b), \eta_{3}(b)\right), b \in B$. 
Define the 1-form $\eta=\eta_{1} d b_{1}+\eta_{2} d b_{2}+\eta_{3} d b_{3}$ on $B$ where $\eta_{i} \in C^{\infty}(B)$ are as in Proposition 3.9.

Proposition 3.10. Let $\mathcal{F}=(X, \omega, f) \in \mathcal{L}(2,2)$. There are local sections $\left(e_{1}, e_{2}, e_{3}\right)$ of $R_{c}^{2} f^{\#} \mathbb{Z}$ such that the period lattice of $\mathcal{F}$ is generated by the 1-forms:

$$
\tau_{1}=\tau_{0}+d H, \quad \tau_{2}=2 \pi d s_{2}, \quad \tau_{3}=d r
$$

where $\tau_{0}=-\log |s| d s_{1}+\operatorname{Arg}(s) d s_{2}$ and $H$ is a smooth function of $b=$ $\left(s_{1}, s_{2}, r\right) \in D \times I$ such that $d H=\eta$. The monodromy of $f$ expressed in terms of $\Lambda=\left\langle\tau_{1}, \tau_{2}, \tau_{3}\right\rangle$ is represented by the matrix: $\left(\begin{array}{lll}1 & 0 & 0 \\ 1 & 1 & 0 \\ 0 & 0 & 1\end{array}\right)$.

Proof. One can construct generators $e_{1}(b), e_{2}(b), e_{3}(b)$ of $H_{1}\left(X_{b}, \mathbb{Z}\right), b \in B_{0}$ by means joining integral curves of $v_{j}$ in a suitable way. For instance, we define a representative of $e_{1}$ to be the ordered composition of paths $\gamma=$ $\left(\gamma_{1}, \gamma_{2}, \gamma_{3}, \tilde{\gamma}_{1}, \tilde{\gamma}_{2}, \tilde{\gamma}_{3}\right)$. Here, $\gamma_{i}$ is an integral curve of $v_{q_{i}}$ starting at a point $x_{i-1}$ running a time $t_{i} \in\left[0, \alpha_{i}\right]$ and finishing at a point $x_{i}$. Similarly, $\tilde{\gamma}_{i}$ is an integral curve of $v_{i}$ starting at a point $\tilde{x}_{i-1}$ running a time $\tilde{t}_{i} \in\left[0, \eta_{i}\right]$ and finishing at a point $\tilde{x}_{i}$. Then, the curve $\gamma$ is determined by the initial condition $x_{0}=\Sigma_{1}(b), \tilde{x}_{0}=x_{3}$. It follows from equations (9) and (10) that $\gamma$ is closed and non-trivial. For constructing a representative of $e_{j}, j=2,3$, we take an integral curve of $v_{j}$ starting at $\Sigma_{1}(b)$ and flowing from time 0 to 1. Now we can use formula (4) to compute the period 1-forms. It follows that $\tau_{1}=\sum \alpha_{j} d b_{j}+\sum \eta_{j} d b_{j}$. Since $\tau_{1}$ and $\tau_{0}=-\log |s| d s_{1}+\operatorname{Arg}(s) d s_{2}$ are closed, then $\tau_{1}=\tau_{0}+d H$ for some $H \in C^{\infty}(B)$. The computation of $\tau_{2}$ and $\tau_{3}$ is direct from (4).

\section{The family $\mathcal{L}(1,2)$}

There is a fairly complete understanding of the class of non-degenerate (Morse-Bott) singularities of integrable Hamiltonian systems (cf. Eliasson [3], Tien-Zung and Miranda [17]). Generically, the function components of the fibration-i.e., the integrals of the system - can be reduced to quadratic polynomials. In contrast, for some special Lagrangian singularities arising from integrable Hamiltonian systems, one should expect cubic terms (cf. Fu $[4])$.

$T^{2}$-symmetric special Lagrangian singularities. Let $X$ be a symplectic 6-manifold and $f:(X, \omega) \rightarrow B$ a Lagrangian fibration which is admissible in the sense of Definition 2.2. Denote by $\omega_{0}=\sum_{i} d x_{i} \wedge d y_{i}$ the standard symplectic form on $\mathbb{C}^{3} \cong \mathbb{R}^{6}$ with canonical coordinates $\left(x_{i}, y_{i}\right), z_{i}=x_{i}+$ $\sqrt{-1} y_{i}$ and let $\Omega_{0}=d z_{1} \wedge d z_{2} \wedge d z_{3}$. 
Definition 4.1. Let $f:(X, \omega) \rightarrow B$ be a Lagrangian fibration and let $p \in \operatorname{Crit}(f)$ and let $k=\left.\operatorname{Rank} f_{*}\right|_{p}$. Let $O_{p}$ denote the Poisson orbit of $p$. We say that $O_{p}$ is a rank $k$ complexity one singularity if there is an open neighbourhood $W \subseteq X$ of $O_{p}$ and a Hamiltonian $T^{2}$ action $\Phi: T^{2} \times W \rightarrow W$ such that $f(\Phi(t, x))=f(x)$ for each $(t, x) \in T^{2} \times W$.

Remark 4.2. Regarding $\left.f\right|_{W}$ as an integrable Hamiltonian system, we can think of $T^{2}$ as a symmetry group of the system. Notice that if $k=0$, i.e., $p$ is a fixed point of the Poisson action, then $p$ is also a fixed point of $\Phi$. It is a standard fact that the Hamiltonian action of a $k$-torus on a symplectic manifold $M$ is completely determined on a neighbourhood of a fixed point $x_{0}$ by the weights of the isotropy representation of the linear action of $T^{k}$ on $T_{x_{0}} M$. These are elements $\rho_{1}\left(x_{0}\right), \ldots \rho_{n}\left(x_{0}\right) \in \mathfrak{t}^{*}=\operatorname{Lie}\left(T^{k}\right)^{*}$ (cf. Guillemin and Sternberg [12]).

Definition 4.3. Let $f:(X, \omega) \rightarrow B$ be a Lagrangian fibration, $p \in \operatorname{Crit}(f)$ and $O_{p} \in X$ a rank $k$ Poisson orbit of $f$. We say that $O_{p}$ is special if there is an open neighbourhood $U \subseteq X$ of $O_{p}$ and canonical coordinates $\left(z_{i}, \bar{z}_{i}\right)$ on $U$ such that $\left.f\right|_{U}$ is special Lagrangian with respect to $\left(\omega_{0}, \Omega_{0}\right)$.

Example 4.4. Let $(X, \omega, f) \in \mathcal{L}(2,2)$ and $p \in \operatorname{Crit}(f)$. Then $O_{p} \subset X$ is a special singularity, which is also a rank one complexity one singularity.

The Harvey-Lawson singularity. We review an example proposed by Harvey and Lawson (cf. [13, §III.3.A]). This provides an example of a special rank zero complexity one singularity.

Let us consider the map $F: \mathbb{C}^{n} \rightarrow \mathbb{R}^{n}$ given by $F=\left(F_{1}, \ldots, F_{n}\right)$ where

$$
F_{1}=\operatorname{Im} \prod z_{i}, \quad F_{k}=\left|z_{1}\right|^{2}-\left|z_{k}\right|^{2}, \quad k=2, \ldots n .
$$

The fibres of $F$ are Lagrangian with respect to the standard symplectic form on $\mathbb{C}^{n}$; for this, one only needs to check that $\left\{F_{i}, F_{j}\right\}=0$ for $i, j=1, \ldots, n$. In other words, $F$ defines an integrable Hamiltonian system. One can also check that $\operatorname{Re}\left(\operatorname{det}_{\mathbb{C}}\left(\partial_{\bar{z}_{j}} F_{i}\right)\right)=0$, hence the fibres of $F$ are special Lagrangian. We observe that the map $\mu:=\left(F_{2}, \ldots, F_{n}\right)$ is the moment map of the Hamiltonian $T^{n-1}$ action on $\mathbb{C}^{n}$ given by:

$$
\left(z_{1}, \ldots, z_{n}\right) \mapsto\left(e^{i \theta_{1}} z_{1}, \ldots, e^{i \theta_{n}} z_{n}\right),
$$

with $\theta_{1}+\cdots+\theta_{n}=0$. This action preserves the fibres of $F$. Now let $x=\left(x_{1}, \ldots, x_{n}\right) \in \mathbb{R}^{n}$ and let $z \in F^{-1}(x)$. Denote by $T \cdot z$ the $T^{n-1}$-orbit of $z$. Then, $T \cdot z$ is homeomorphic to $T^{n-1}$ unless $z \in \operatorname{Crit}(F)=\bigcup_{1 \leq i<j \leq n} P_{i j}$ where,

$$
P_{i j}=\left\{\left(z_{1}, \ldots, z_{n}\right) \in \mathbb{C}^{n} \mid z_{i}=z_{j}=0\right\} .
$$

For $z \in \operatorname{Crit}(F)$, the orbit $T \cdot z$ is a torus of lower dimension and it is a point when $z=0$. A fibre $F^{-1}(x)$ disjoint from Crit $(F)$ is homeomorphic 
to $T^{n-1} \times \mathbb{R}$ and for $x \in \Delta:=F($ Crit $(F))$ the fibre $F^{-1}(x)$ is a singular fibre.

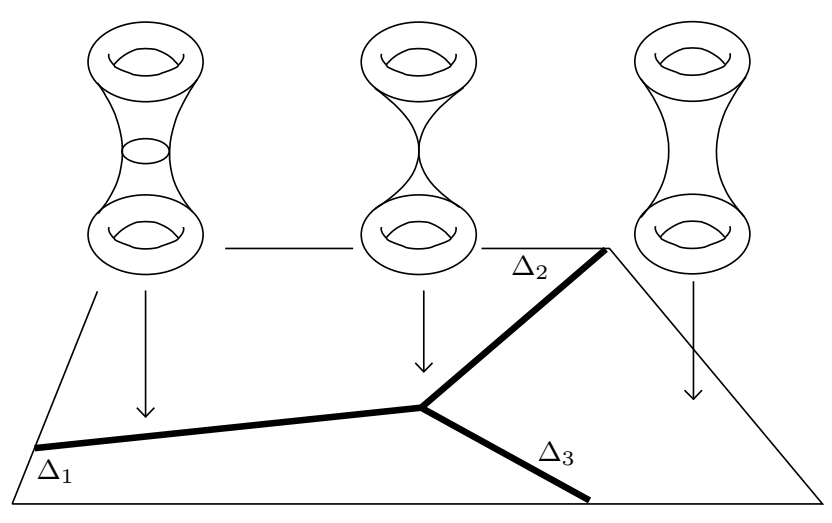

Figure 2. The fibres of $F$.

For $n=3, \Delta=\Delta_{1} \cup \Delta_{2} \cup \Delta_{3} \cup\{0\}$ where $\Delta_{1}=\left\{x_{1}=0, x_{2}=x_{3}>0\right\}$, $\Delta_{j}=\left\{x_{1}=x_{j}=0, x_{j}<0\right\}$, for $j=2,3$. The fibre over $x \in \Delta_{i}$ is homeomorphic to

$$
S^{1} \times\left[\mathbb{R} \times S^{1} /\left(\{\text { point }\} \times S^{1}\right)\right],
$$

whereas the fibre over $0 \in \mathbb{R}^{3}$ is homeomorphic to

$$
\mathbb{R} \times T^{2} /\left(\{\text { point }\} \times T^{2}\right)
$$

In particular, we conclude that the map $F$ is not proper.

Remark 4.5. Joyce observed (cf. $[\mathbf{1 4}, \S 5]$ and $[\mathbf{1 5}, \S 4]$ ) that, in three dimensions, any connected special Lagrangian 3-manifold in $\mathbb{C}^{3}$ which is invariant under the above $T^{2}$-action is a subset of some fibre of the map (11).

The topological $(1,2)$ fibre. We outline Gross' construction of a topological 3-torus fibration with fibre of type $(1,2)$. For the details we refer the reader to [8, Example 2.10].

Construction 4.6 (Gross $[8]$ ). Let $B=B^{3}$ be a 3 -ball. We define $\Delta \subset B$ a cone over three points as follows. Identify $B \backslash\{0\}$ with $S^{2} \times(0,1)$ and let $p_{1}, p_{2}, p_{3} \in S^{2}$. Define $\Delta_{i}=\left\{p_{i}\right\} \times(0,1)$. These are the "legs" of the cone. Define $\Delta=\Delta_{1} \cup \Delta_{2} \cup \Delta_{3} \cup\{0\}$, where $\{0\}$ is the vertex of the cone.

Let $Y=S^{1} \times B$ and $Y^{\prime}=Y \backslash(\{p\} \times \Delta)$, where $p \in S^{1}$. Let $L \cong \mathbb{Z}^{2}$ and define $T(L)=L \otimes_{\mathbb{Z}} \mathbb{R} / L$. Now consider a principal $T(L)$-bundle $\pi^{\prime}: X^{\prime} \rightarrow Y^{\prime}$ with Chern class $c_{1} \in H^{2}\left(Y^{\prime}, L\right)$. Then the class $c_{1}$ is represented by a triple $\left(a_{1}, a_{2}, a_{3}\right)$ where $a_{i} \in L$. It is shown (cf. [8, Ex. 2.10]) that by choosing 
$c_{1}=((1,0),(0,1),(-1,-1))$ there is a unique manifold $X$ such that $X^{\prime} \subset X$ and a commutative diagram of smooth maps:

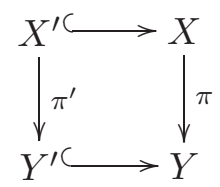

such that $\pi$ is proper. Furthermore, it is shown that in a neighbourhood $U \cong \mathbb{C} \times \mathbb{R}^{2} \subset Y$ of the vertex of $\Delta$, the map $\pi: \pi^{-1}(U) \rightarrow U$ coincides with the map $\tilde{\pi}: \mathbb{C}^{3} \rightarrow \mathbb{C} \times \mathbb{R}^{2}$ given by:

$$
\tilde{\pi}\left(z_{1}, z_{2}, z_{3}\right)=\left(z_{1} z_{2} z_{3},\left|z_{1}\right|^{2}-\left|z_{2}\right|^{2},\left|z_{1}\right|^{2}-\left|z_{3}\right|^{2}\right) .
$$

Now define $f: X \rightarrow B$ to be the composition of $\pi: X \rightarrow Y$ with the projection $Y \rightarrow B$. Then, $f$ is a continuous map whose fibre over $b \in B \backslash \Delta$ is $T^{3}$. The fibre over $b \in \Delta_{i}$ is homeomorphic to $S^{1} \times\left[S^{1} \times S^{1} /\left(\{\right.\right.$ point $\left.\left.\} \times S^{1}\right)\right]$, i.e., it is a $(2,2)$ fibre, whereas the fibre over the vertex of $\Delta$ is homeomorphic to $S^{1} \times T^{2} /\left(\{\right.$ point $\left.\} \times T^{2}\right)$, i.e., it is a $(1,2)$ fibre.

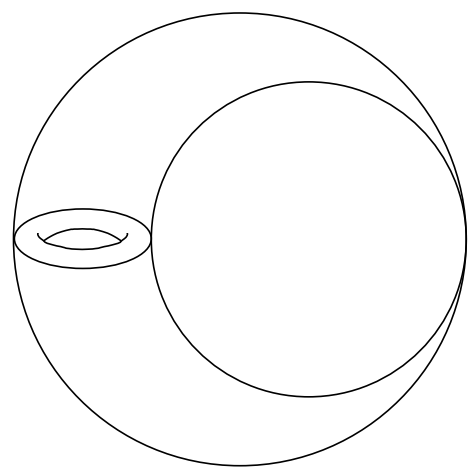

Figure 3. Singular fibre of type $(1,2)$.

It turns out that the $T(L)$ action on $X^{\prime}$ action extends to $X$, moreover, $\operatorname{Crit}(f) \subset X$ consists of the union of the critical orbits of this action. There is a single fixed point $p \in \operatorname{Crit}(f)$, which is singular point of $f^{-1}(0)$.

The symplectic structure. Let $f: X \rightarrow B$ as in Construction 4.6 and suppose there is a symplectic structure $\omega$ making $f$ Lagrangian, i.e., defining a triple $(X, \omega, f) \in \mathcal{L}(1,2)$. Furthermore, assume the extended $T(L)$ action on $X$ preserves $\omega$. It is follows from these hypotheses that $p$ is a rank zero complexity one singularity. Let $\mathfrak{t}=\operatorname{Lie}(T(L))$. Then we can regard $L \hookrightarrow \mathfrak{t}$ and identify $c_{1}$ with the isotropy data $\left(\rho_{1}(p), \rho_{2}(p), \rho_{3}(p)\right)$ of the $T(L)$-action at $p$. 
Theorem 4.7. Let $f:(X, \omega) \rightarrow B$ be a Lagrangian fibration of type $(1,2)$. Assume there is a fibre-preserving $T:=T(L)$ action preserving $\omega$. Let $p \in \operatorname{Crit}(f) \cap f^{-1}(0)$. Then there is an open neighbourhood $U \subset X$ of $p$, a symplectomorphism $\psi: U \rightarrow V \subseteq\left(T_{p} X, \omega_{0}\right)$ and a diffeomorphism $\varphi: f(U) \rightarrow \mathbb{R}^{3}$ such that $q \circ \psi=\left.\varphi \circ f\right|_{U}$, where $q: V \rightarrow \mathbb{R}^{3}$ is a Lagrangian fibration given by $q=\left(h,\left|z_{1}\right|^{2}-\left|z_{2}\right|^{2},\left|z_{1}\right|^{2}-\left|z_{3}\right|^{2}\right), h \in C^{\infty}(V)$. Furthermore, if $p$ is special, then $h=\operatorname{Im} z_{1} z_{2} z_{3}$.

Proof. Consider $\mu: X \rightarrow \mathfrak{t}^{*}$ the moment map of the $T$ action around $p$. According to [12], there is a neighbourhood $U \subseteq X$ of $p$ and an equivariant symplectomorphism $\psi: U \rightarrow V \subseteq\left(T_{p} X, \omega_{0}\right)$ such that $\mu=\psi^{*} M$, where $M=c+\sum_{i} \rho_{i}\left|z_{i}\right|^{2}, c \in \mathfrak{t}^{*}$. Without loss of generality we can assume $c=0$ and choose a basis of $\mathfrak{t}^{*}$ such that $\rho_{1}=(1,1), \rho_{2}=(-1,0)$ and $\rho_{3}=(0,-1)$. Then we can write $M=\left(M_{1}, M_{2}\right)$, where $M_{j}=\left|z_{1}\right|^{2}-\left|z_{j}\right|^{2}$. Let $v_{j}$ be the vector field on $V$ determined by the equation: $\iota\left(v_{j}\right) \omega_{0}=d M_{j}$. The orbits of $v_{j}$ are periodic with period $2 \pi$. Now let $\Sigma$ be a section of $f$ over $B^{\prime}:=f(U)$ such that $\Sigma\left(B^{\prime}\right) \subset U \backslash \operatorname{Crit}(f)$. Let $y(b)=\psi(\Sigma(b))$ and $g_{j}:[0,2 \pi] \rightarrow V$ be an integral orbit of $v_{j}$ passing through $y(b)$. Then $g_{j}$ pulls back to a loop $\gamma_{j}(b) \subset f^{-1}(b) \cap U$, disjoint from Crit $(f)$. We can assume there is a 1-form $\sigma$ such that $d \sigma=-\omega$. Let $\mathcal{A}_{j}(b)=\int_{\gamma_{j}(b)} \sigma$. One can verify that $\left.\mathcal{A}_{j} \circ f\right|_{U}=$ $M_{j} \circ \psi$. Now let $\alpha$ be a Lagrangian section of $T_{B^{\prime}}^{*}$ close to the zero section. We can choose $\alpha$ such that $\alpha(0) \wedge d \mathcal{A}_{1}(0) \wedge d \mathcal{A}_{2}(0) \neq 0$. Then there is an open neighbourhood of $0 \in B^{\prime}$ in which $\alpha \wedge d \mathcal{A}_{1} \wedge d \mathcal{A}_{2} \neq 0$ and a unique smooth function $\mathcal{A}$ such that $\mathcal{A}(0)=0$ and $d \mathcal{A}=\alpha$. Then $\varphi=\left(\mathcal{A}, \mathcal{A}_{1}, \mathcal{A}_{2}\right)$ defines a diffeomorphism from a small neighbourhood of $0 \in \mathbb{R}^{3}$ denoted, with abuse of notation by $B$, into $\mathbb{R}^{3}, \varphi: B \rightarrow \varphi(B) \subseteq \mathbb{R}^{3}$. Let $h=\mathcal{A} \circ f \circ \psi^{-1}$. Then $h$ is a $T$-invariant function on $V$ hence $q:=\left(h, M_{1}, M_{2}\right)$ defines a Lagrangian fibration on $V$ such that $q=\left.\varphi \circ f\right|_{U} \circ \psi^{-1}$. Now we can think of $\psi$ as identifying $U \cong V \subseteq \mathbb{C}^{3}$ such that $\left.\varphi \circ f\right|_{U}=\left(h, M_{1}, M_{2}\right)$. In view of Remark 4.5 and since $\psi(\operatorname{Crit}(f))=\operatorname{Crit}(q)=\operatorname{Crit}(M)=\bigcup_{i j}\left\{z_{i}=z_{j}=0\right\}$, we see that if $\left.\varphi \circ f\right|_{U}$ is special Lagrangian then there should exist a 1-form $\alpha$ with the above properties and such that $h=\operatorname{Im} z_{1} z_{2} z_{3}$.

Remark 4.8. Observe that the $T(L)$-action on $X$ can always be assumed to be Hamiltonian. Indeed, the above action is chosen so that $f$ has the desired monodromy, in particular, it induces monodromy invariant cycles $e_{1}(b), e_{2}(b) \in H_{1}\left(f^{-1}(b), \mathbb{Z}\right)$ which can be used to compute the action integrals $\mathcal{A}_{e_{1}}, \mathcal{A}_{e_{2}}$. Then $\mu_{i}=\mathcal{A}_{i} \circ f$ define the moment map $\left(\mu_{1}, \mu_{2}\right)$ of a $T=S^{1} \times S^{1}$ action, which is defined on $X$ as $e_{i}$ are monodromy invariant. It is a consequence of $\left[8\right.$, Prop. 3.3] and [7, Thm. 2.2] that $p \in f^{-1}(0) \cap$ Crit $(f)$ can be made into a special singularity with respect to $\left(\omega_{0}, \Omega_{0}\right)$.

Corollary 4.9. Let $(X, \omega, f) \in \mathcal{L}(1,2)$. Let $p \in f^{-1}(0) \cap \operatorname{Crit}(f)$ and let $\left(T_{p} X, \omega_{0}\right)$, where $\omega_{0}=\frac{i}{2} \sum d z_{j} \wedge d \bar{z}_{j}$. There is a neighbourhood $U \subset X$ of 
$p$ and a 3-ball $B$ centred at $0 \in \mathbb{R}^{3}$ such that $f(U)=B$, a diffeomorphism $\varphi: B \rightarrow \varphi(B) \subseteq \mathbb{R}^{3}$ and a symplectomorphism $\psi: U \rightarrow V \subseteq\left(T_{p} X, \omega_{0}\right)$ such that $\left.\varphi \circ f\right|_{U}=F \circ \psi$ where $F\left(z_{1}, z_{2}, z_{3}\right)=\left(\operatorname{Im} z_{1} z_{2} z_{3},\left|z_{1}\right|^{2}-\left|z_{2}\right|^{2},\left|z_{1}\right|^{2}-\left|z_{3}\right|^{2}\right)$.

Example of a Lagrangian fibration of type (1,2). Here we show that the family $\mathcal{L}(1,2)$ is not void. We construct a Lagrangian fibration of type $(1,2)$ for each $H \in C^{\infty}(B), B \subset \mathbb{R}^{3}$ an open ball. The arguments we use here are valid in any dimension.

Consider the map $F: \mathbb{C}^{n} \rightarrow \mathbb{R}^{n}$, where $F=\left(F_{1}, \ldots, F_{n}\right)$ as in (11). The quotient $\mathbb{C}^{n} / T^{n-1}$ can be identified with $\mathbb{C} \times \mathbb{R}^{n-1}$ by means of the map $\pi: \mathbb{C}^{n} \rightarrow \mathbb{C} \times \mathbb{R}^{n-1}$,

$$
\pi(z)=\left(\prod z_{i},\left|z_{1}\right|^{2}-\left|z_{2}\right|^{2}, \ldots,\left|z_{1}\right|^{2}-\left|z_{n}\right|^{2}\right) .
$$

Let $\prod z_{i}=u+\sqrt{-1} b_{1} \in \mathbb{C}$ and $b_{j}=\left|z_{1}\right|^{2}-\left|z_{j}\right|^{2}$ and $b=\left(b_{1}, \ldots b_{n}\right)$. Letting $x_{i}=\left|z_{i}\right|^{2}$ the following relations hold:

$$
\left\{\begin{array}{l}
\prod_{i=1}^{n} x_{i}=u^{2}+b_{1}^{2} \\
x_{1}-x_{j}=b_{j}, \quad j \geq 2 .
\end{array}\right.
$$

We can restate these equations (renaming $x:=x_{1}$ ) as:

$$
x \prod_{j \geq 2}\left(x-b_{j}\right)-b_{1}^{2}=u^{2} .
$$

Define $P_{b}(x)=x \prod_{j \geq 2}\left(x-b_{j}\right)-b_{1}^{2}$. We can regard $P_{b}(x)$ as a polynomial in the variable $x$ with $b \in \mathbb{R}^{n}$ acting as a parameter. We notice that for all values of $b, P_{b}(x)=0$ has always a non-negative real solution. Define $z_{b}^{\mathbb{R}}=\left\{\zeta(b) \in \mathbb{R} \mid P_{b}(\zeta(b))=0\right\}$. This is an ordered set, so we can take $\zeta_{0}(b)=\max Z_{b}^{\mathbb{R}}$. We observe that $P_{b}(x)>0$ for $x>\zeta_{0}(b) ; P_{b}^{\prime}(x) \neq 0$ for $x>\zeta_{0}(b)$ and $P_{b}^{\prime}\left(\zeta_{0}(b)\right)=0$ if and only if $b \in \Delta$. Observe that $\zeta_{0}(b)$ becomes a multiple root of $P_{b}(x)$ when $b \in \Delta$.

Lemma 4.10. The function $\zeta_{0}(b)$ is smooth on $\mathbb{R}^{n} \backslash \Delta$ and continuous on $\Delta$. Let $\partial_{J_{k}}^{k} \zeta_{0}$ denote an order $k$ partial derivative of $\zeta_{0}, J_{k}=j_{1}, \ldots, j_{n}$, $j_{1}+\cdots+j_{n}=k$. Let $B \subset \mathbb{R}^{n}$ be a small neighbourhood of $0 \in \mathbb{R}^{n}$. Then,

$$
\partial_{J_{k}}^{k} \zeta_{0}=\sum_{l<\infty} \frac{\left.G_{l}(b, x)\right|_{\zeta_{0}}}{\left(P_{b}^{\prime}\left(\zeta_{0}\right)\right)^{\lambda_{l}}},
$$

where $G_{l}(b, x)$ is bounded on $B$ and $\lambda_{l} \in \mathbb{Z}^{+}$is a finite power.

Proof. For $b \in \mathbb{R}^{n} \backslash \Delta, P_{b}^{\prime}\left(\zeta_{0}(b)\right) \neq 0$ and it follows that $\zeta_{0}(b)$ is smooth on $\mathbb{R}^{n} \backslash \Delta$. Let $G(b)=P_{b}\left(\zeta_{0}(b)\right)$ and consider $\partial_{b_{j}} G$. We notice that $G \equiv 0$ on $\mathbb{R}^{n}$, hence $\partial_{b_{j}} G \equiv 0$. This implies

$$
\partial_{b_{j}} \zeta_{0}=-\frac{\left.\partial_{b_{j}} P_{b}\right|_{\zeta_{0}}}{P_{b}^{\prime}\left(\zeta_{0}\right)}
$$


The function $G_{1_{j}}(b)=\left.\partial_{b_{j}} P_{b}\right|_{\zeta_{0}}$ is bounded on a small ball $B \subset \mathbb{R}^{n}$ centred at $0 \in \mathbb{R}^{n}$. The verification of the case $k>1$ is left to the reader.

Let $\epsilon>0$ and let $\zeta_{\epsilon}(b)$ be the maximal real solution of $P_{b}(x)-\epsilon^{2}=0$. Observe that $\zeta_{0}(b)<\zeta_{\epsilon}(b)$ and $P_{b}^{\prime}\left(\zeta_{\epsilon}(b)\right) \neq 0$ for all $b \in \mathbb{R}^{n}$. It is easy to verify that $\zeta_{\epsilon}(b)$ is a smooth function on $\mathbb{R}^{n}$.

Corollary 4.11. Let $F: \mathbb{C}^{n} \rightarrow \mathbb{R}^{3}$ as in (11). Let $\zeta_{0}$ and $\zeta_{1}$ be the maximal real solutions of $P_{b}(x)=0$ and $P_{b}(x)-1=0$ respectively. Let $\theta_{ \pm}(b)=$ $\operatorname{Arg}\left( \pm 1+i b_{1}\right)$. The maps $\Sigma^{-}$and $\Sigma^{+}$,

$$
\Sigma^{ \pm}(b)=\left(\sqrt{\zeta_{1}(b)} \cdot e^{i \theta_{ \pm}(b)}, \sqrt{\zeta_{1}(b)-b_{2}}, \ldots, \sqrt{\zeta_{1}(b)-b_{n}}\right)
$$

are sections of $F$ which are smooth on $\mathbb{R}^{n}$. Let $\theta_{0}(b)=\operatorname{Arg}\left(i b_{1}\right)$. The section

$$
\Sigma^{0}(b)=\left(\sqrt{\zeta_{0}(b)} \cdot e^{i \theta_{0}(b)}, \sqrt{\zeta_{0}(b)-b_{2}}, \ldots, \sqrt{\zeta_{0}(b)-b_{n}}\right)
$$

is smooth on $\mathbb{R}^{n} \backslash \Delta$ and continuous on $\mathbb{R}^{n}$.

Proof. It remains to verify that the above maps are sections of $F$. Let $\pi$ as in (14). A direct computation shows that $\pi\left(\Sigma^{ \pm}(b)\right)=\left( \pm 1+i b_{1}, b_{2}, \cdots, b_{n}\right)$ and $\pi\left(\Sigma^{0}(b)\right)=\left(i b_{1}, b_{2}, \ldots, b_{n}\right)$. Since $F$ factors via $\pi$ in an obvious way, the claim follows.

Now let $\phi_{i}^{t}$ be the flow of the Hamiltonian vector field $V_{F_{i}}$ and consider the Poisson $\mathbb{R}^{n}$-action, $\Phi: \mathbb{R}^{n} \times \mathbb{C}^{n} \rightarrow \mathbb{C}^{n}$ :

$$
\Phi\left(t_{1}, \ldots, t_{n} ; z\right)=\phi_{1}^{t_{1}} \circ \cdots \circ \phi_{n}^{t_{n}}(z) .
$$

Remark 4.12. Observe that $\Phi$ is free and transitive along the fibres of $F$ over $\mathbb{R}^{n} \backslash \Delta$. Let $b_{0} \in \mathbb{R}^{n} \backslash \Delta$. Then, for each $z=\Sigma^{-}\left(b_{0}\right)$ there is $\left(\alpha_{1}^{0}, \ldots, \alpha_{n}^{0}\right) \in \mathbb{R}^{n}$ such that $\Phi\left(\alpha_{1}^{0}, \ldots, \alpha_{n}^{0} ; z\right) \in \Sigma^{+}\left(b_{0}\right)$. It follows from similar arguments to the ones used in the proof of Lemma 3.9 that there are locally defined $C^{\infty}$ functions $\alpha_{i}(b)$ on $\mathbb{R}^{n} \backslash \Delta$ such that $\alpha_{i}\left(b_{0}\right)=\alpha_{i}^{0}$ and such that $\Phi\left(\alpha_{1}(b), \ldots, \alpha_{n}(b), z\right) \in \Sigma^{+}\left(\mathbb{R}^{n} \backslash \Delta\right)$ for all $z \in \Sigma^{-}\left(\mathbb{R}^{n} \backslash \Delta\right)$.

Denote by $\alpha:=\alpha_{1}$ and $\phi^{t}$ the flow of $V_{F_{1}}$. Let $\mathcal{O}^{-}(b)$ and $\mathcal{O}^{+}(b)$ the $T^{n-1}$ orbits of $\Sigma^{-}(b)$ and $\Sigma^{+}(b)$ respectively; it follows that $\mathcal{O}^{-}(b) \cong \mathcal{O}^{+}(b) \cong$ $T^{n-1}$.

It is easy to see that for $x \in \mathcal{O}^{-}(b), \phi^{\alpha(b)}(x) \in \mathcal{O}^{+}(b)$. Let $z(b) \in \mathcal{O}^{-}(b)$ and $w(b) \in \mathcal{O}^{+}(b)$. Let $\varphi$ denote the flow of $\pi_{*}\left(V_{F_{1}}\right)$. It is straightforward to check that the solution to the equation $\varphi^{t(b)}(\pi(z(b)))=\pi(w(b))$ is precisely $t(b)=\alpha(b)$. We want to find an explicit expression of $\alpha(b)$. An easy computation shows that $\pi_{*}\left(V_{F_{1}}\right)=-\chi \partial_{u}$ where,

$$
\chi=\sum_{j=1}^{n} \frac{\prod_{i=1}^{n}\left|z_{i}\right|^{2}}{\left|z_{j}\right|^{2}} .
$$


Using formulae (15), we see that $\chi=\partial_{x} P_{b}(x)$. Regarding $\mathbb{C} \times \mathbb{R}^{n-1}=\mathbb{R}^{n+1}$ with coordinates $(u, b)$, we can write $\pi_{*}\left(V_{F_{1}}\right)$ as the vector field in $\mathbb{R}^{n+1}$ :

$$
-2 u \partial_{x} u \frac{\partial}{\partial u}
$$

Observe that for $b \in \mathbb{R}^{n} \backslash \Delta$ this vector field is not singular.

Lemma 4.13. Let $V$ be a vector field over $\mathbb{R}$. Let $p_{0}$ and $p$ be two points in $\mathbb{R}$ and assume $V(u) \neq 0$ for $u \in\left[p_{0}, p\right]$. The time it takes to flow from $p_{0}$ to $p$ is:

$$
T=\int_{p_{0}}^{p} \frac{d u}{V(u)} .
$$

Proof. Let $\varphi(t, u)$ be the flow of $V$. We want to find the time $T=T(p)$ such that $\varphi\left(T, p_{0}\right)=p$. We point out that $\left.\partial_{t} \varphi\left(t, p_{0}\right)\right|_{t=T(u)}=V(u)$. Then the derivative of $\varphi\left(T(u), p_{0}\right)$ with respect to $u$ is $V(u) \partial_{u} T(u)=1$. The claim follows easily from this.

Proposition 4.14. The function $\alpha$ is hypergeometric. Explicitly,

$$
\alpha(b)=-\int_{\zeta_{0}(b)}^{\zeta_{1}(b)} \frac{d x}{\sqrt{P_{b}(x)}}, \quad b \in \mathbb{R}^{n} \backslash \Delta,
$$

where $\zeta_{0}(b)$ is the maximal real root of $P_{b}(x)=x\left(x-b_{2}\right) \cdots\left(x-b_{n}\right)-b_{1}^{2}$ and $\zeta_{1}(b)$ is the maximal real solution of $P_{b}(x)-1=0$.

Proof. First observe that $\pi(z(b))=(-1, b)$ and $\pi(w(b))=(1, b)$. It follows from Lemma 4.13 that:

$$
-\alpha(b)=\int_{(-1, b)}^{(1, b)} \frac{d u}{2 u \partial_{x} u} .
$$

Bearing in mind that $u= \pm \sqrt{P_{b}(x)}$, it is not difficult to see that $\alpha$ is as claimed.

Of course, we can integrate $\alpha$ explicitly only when $n=2$. We are particularly interested in the case when $n \geq 3$, for which we need a precise understanding of the behaviour of $\alpha(b)$ as $b \rightarrow \Delta$. Let us write,

$$
P_{b}=\left(x-\zeta_{0}\right) Q_{b}(x),
$$

where $Q_{b}(x)$ is a polynomial with real coefficients. We notice that $\zeta_{0}(b)$ becomes a (possible multiple) root of $Q_{b}(x)$ if and only if $b \in \Delta$.

Proposition 4.15. Let $\alpha$ as above and let $\partial_{J_{k}}^{k} \alpha$ denote a partial derivative of order $k$. Then, $\alpha$ is bounded from above by

$$
-\frac{2}{\left(Q_{b}\left(\zeta_{0}\right)\right)^{\frac{1}{2}}} \text {. }
$$


There are finite powers $w_{0}, w_{1}, \ldots, w_{n-1} \in \mathbb{Z}^{+}$, depending on $J_{k}$, such that near $\Delta$,

$$
\left|\partial_{J_{k}}^{k} \alpha\right| \simeq \frac{1}{P_{b}^{\prime}\left(\zeta_{0}\right)^{w_{0}}\left|\zeta_{0}-\beta_{1}\right|^{w_{1}} \cdots\left|\zeta_{0}-\beta_{n-1}\right|^{w_{n-1}}},
$$

where $\beta_{i}(b)=\operatorname{Re} \rho_{i}(b)$ are the real part of the roots of $Q_{b}, P_{b}=\left(x-\zeta_{0}\right) Q_{b}(x)$.

Proof. The proof involves the use of a (truncated) asymptotic expansion of $\alpha$. Since the integration limits of $\alpha$ depend on $b$, the estimates of $\partial_{J_{k}}^{k} \alpha$ turn out to be rather messy, as they involve the derivatives of $\zeta_{0}$. Here we estimate $\alpha$ to order $k=0$ and refer the reader to [1] for the details concerning $k \geq 1$.

Let $I=\alpha$ and let $f=Q_{b}^{-\frac{1}{2}}$ and $d g=\left(x-\zeta_{0}\right)^{-\frac{1}{2}} d x$. Integrating $I=\int f d g$ by parts we get,

$$
I=\left.2 \frac{\left(x-\zeta_{0}\right)^{\frac{1}{2}}}{Q_{b}(x)^{\frac{1}{2}}}\right|_{\zeta_{0}} ^{\zeta_{1}}-\int_{\zeta_{0}}^{\zeta_{1}}-\frac{\left(x-\zeta_{0}\right)^{\frac{1}{2}} Q_{b}^{\prime}(x) d x}{\left(Q_{b}(x)\right)^{1+\frac{1}{2}}} .
$$

Let $R_{1}$ be the first summand on (26), and let $I_{1}$ be the integral. We notice that $R_{1}=2\left(Q_{b}\left(\zeta_{1}\right)\right)^{-\frac{1}{2}}$. Since $x$ is such that $0 \leq x-\zeta_{0}(b) \leq 1$, then

$$
0 \geq I_{1} \geq \int_{\zeta_{0}}^{\zeta_{1}}-\frac{Q_{b}^{\prime}(x) d x}{\left(Q_{b}(x)\right)^{1+\frac{1}{2}}}=2\left[\left(Q_{b}\left(\zeta_{1}\right)\right)^{-\frac{1}{2}}-\left(Q_{b}\left(\zeta_{0}\right)\right)^{-\frac{1}{2}}\right]
$$

Then we get, $|I| \simeq 2\left(Q_{b}\left(\zeta_{0}\right)\right)^{-\frac{1}{2}}$.

Remark 4.16. What Proposition 4.15 says is that the derivatives of $\alpha$ blow up at $\Delta$ when the $\zeta_{0}$ becomes a multiple root of $Q_{b}(x)$. Furthermore, $\alpha$ and all its derivatives are bounded by a rational function having a pole of certain finite order along $\Delta$. For instance, when $n=3, \zeta_{0}$ becomes a root of $Q_{b}(x)$ as $b$ approaches to the spokes of $\Delta$, so $\alpha$ blows up there with order at most $-\frac{1}{2}$. This root becomes double at $0 \in \mathbb{R}^{3}$, so $\alpha$ blows up there with order at most -1 .

Now let $B \subseteq \mathbb{R}^{n}$ be an open neighbourhood of $0 \in \mathbb{R}^{n}$, let $B_{0}:=B \backslash \Delta$ and let $\alpha_{1}, \ldots, \alpha_{n}$ as in Remark 4.12. Define a map $A: \Sigma^{-}\left(B_{0}\right) \rightarrow \Sigma^{+}\left(B_{0}\right)$ as $A(z)=\Phi\left(\alpha_{1}, \ldots, \alpha_{n} ; z\right)$. In view of (18), we can write $A$ explicitly as $A$ : $\left(z_{1}, z_{2}, \ldots, z_{n}\right) \mapsto\left(-\bar{z}_{1}, z_{2}, \ldots z_{n}\right)$. We verify that $A$ is smooth, furthermore, $A$ extends smoothly to $z \in \Sigma^{-}(B)$, regardless of the fact that the Poisson action is not freely transitive over singular fibres.

Let $\tau_{0}=\sum \alpha_{j} d b_{j}$. We can find a 1 -form $\eta=\sum \eta_{j} d b_{j}$ on $B$ such that $\tau:=\tau_{0}+\eta$ is closed. Indeed, let $\sigma$ be such that $d \sigma=\omega$ and let $\gamma(b)$ be a curve joining $\Sigma^{-}(b)$, and $\Sigma^{+}(b)$. One can verify that $\tau_{0}=d A_{\gamma}+R_{\gamma}$ where 
$A_{\gamma}=\int_{\gamma} \sigma$ and $R_{\gamma}$ is a 1 -form (c.f. (5)). Defining $\eta=d H-R_{\gamma}$ for any $H \in C^{\infty}(B)$, we obtain $\tau=d\left(A_{\gamma}+H\right)$.

Let $A^{\prime}: \Sigma^{+}(B) \rightarrow \Sigma(B):=A^{\prime}\left(\Sigma^{+}(B)\right)$ be the map, $A^{\prime}(z)=\Phi\left(\eta_{1}, \ldots\right.$, $\left.\eta_{n} ; z\right)$. The composition $Q=A^{\prime} \circ A$,

$$
Q: \Sigma^{-}(B) \rightarrow \Sigma(B) .
$$

is a $C^{\infty}$ map.

Proposition 4.17. Let $\tau=\tau_{0}+\eta$ be the 1-form as in the paragraph above. Then, there is a symplectic manifold $(X, \omega)$ and a Lagrangian fibration $f$ : $X \rightarrow B$ such that $\tau_{1}:=\tau$, and $\tau_{j}=\pi d b_{j}, j=1, \ldots n$, are the period 1 -forms of $f$. Furthermore, when $n=3, f$ coincides topologically with the example in Construction 4.6.

Proof. We saw in Proposition 4.15 that the function $\alpha_{1}(b)$ is bounded from above by $-2\left(Q_{b}\left(\zeta_{0}(b)\right)\right)^{-\frac{1}{2}}$. We can find a smaller neighbourhood $B^{\prime} \subseteq B$ of $\Delta$ such that $\alpha_{1}(b)+\eta_{1}(b)<0$ for $b \in B^{\prime}$. Let $B_{0}^{\prime}=B^{\prime} \backslash \Delta$. Now let $\mathcal{O}_{b}$ be the subset of $F^{-1}(b)$ defined by:

$$
\mathcal{O}_{b}:=\left\{\Phi(t ; z) \mid t \in\left[\alpha_{1}(b)+\eta_{1}(b), 0\right] \times[0,2 \pi] \cdots \times[0,2 \pi] \subseteq \mathbb{R}^{n}\right\} .
$$

Let $\bar{U}=\overline{\bigcup_{b \in B^{\prime}} \mathcal{O}_{b}}$, this is a $T^{n-1}$-invariant subset of $\mathbb{C}^{n}$. We see that for $b \in B_{0}^{\prime}, \bar{U} \cap F^{-1}(c)$ is a bounded cylinder and for $b \in \Delta$ it is a bounded set in $F^{-1}(b)$. In both cases, the boundary of these sets are the $T^{n-1}$-orbits: $\mathcal{T}^{-}(b)=T \cdot \Sigma^{-}(b)$ and $\mathcal{T}(b)=T \cdot \Sigma(b)$.

Now let $W \subset F^{-1}\left(B^{\prime}\right)$ be a small $T^{n-1}$-invariant neighbourhood of $\Sigma^{-}\left(B^{\prime}\right)$ such that $W \cap \operatorname{Crit}(F)=\emptyset$. Let $x \in W$ such that $F(x)=b$. There is a finite $t \in \mathbb{R}^{n}, t=t(x)$, such that $x=\Phi\left(t, \Sigma^{-}(b)\right)$. Let $Q: \Sigma^{-}\left(B^{\prime}\right) \rightarrow \Sigma\left(B^{\prime}\right)$ as in (28). Define a map $\mathcal{Q}: W \rightarrow F^{-1}\left(B^{\prime}\right), \mathcal{Q}(x)=y=\Phi(t(x), \Sigma(b))$. It follows that $Q$ extends $Q$. Moreover, similar arguments to the ones used in Lemma 3.9 can be used to show that $t(x)$ is $C^{\infty}$. Let $W^{\prime}:=\mathcal{Q}(W)$. Then, $\mathcal{Q}: W \rightarrow W^{\prime}$ is clearly invertible, moreover, $\mathcal{Q}$ is a diffeomorphism and $\mathcal{Q}$ sends $\mathcal{T}^{-}$diffeomorphically to $\mathcal{T}$.

Now let $\mathcal{U}=W \cup \bar{U} \cup W^{\prime}$ and let $x, y \in \mathcal{U}$. We define $X=\mathcal{U} / \sim$ where $x \sim y \Leftrightarrow$ either $x=y$ or $y=Q(x)$ if $x \in W$ and $y \in W^{\prime}$. By means of this identification, $X$ is a smooth manifold. Intuitively, $Q$ identifies the two components on the boundary of $\bar{U}$. F induces a smooth map $f: X \rightarrow B^{\prime}$ such that $\left.f\right|_{V}=\left.F\right|_{V}$ on a neighbourhood $V \subset$ int $\bar{U}$ of $\operatorname{Crit}(f)$. If we can make $X$ into a symplectic manifold, then the periods of $X$ are, by construction, $\tau_{1}, \ldots, \tau_{n}$.

Now let $\omega$ be the standard symplectic structure on $\mathbb{C}^{n}$. Then $\omega$ restricts to symplectic forms on $W$ and $W^{\prime}$. Let us consider 1 -form $\tau:=\tau_{1}$ which may be multi-valued on $B_{0}^{\prime}$. We can choose a domain $D \subset B_{0}^{\prime}$ where $\tau$ is single valued. Let $V_{\tau}$ the vector field determined by the equation $F^{*} \tau=\iota\left(V_{\tau}\right) \omega$. 
Since $\tau$ is closed, $V_{\tau}$ is a symplectic vector field, i.e., its flow, $\psi_{s}$, defines a 1parameter family of symplectomorphisms. In particular, its time $s=1$ flow map, $\psi_{1}$, is a symplectomorphism. One can easily check that $\left.\psi_{1}\right|_{W \cap F^{-1}(D)}=$ $\left.\mathcal{Q}\right|_{W \cap F^{-1}(D)}$. This implies that $\mathcal{Q}$ extends $\psi_{1}$ to $W$, in particular, $\mathcal{Q}^{*} \omega$ and $\omega$ coincide on $W \cap F^{-1}(D)$. It follows that $Q^{*} \omega$ and $\omega$ coincide on $W \backslash\left\{f^{-1}(\Delta) \cap\right.$ $W\} \subset W$ which is dense in $W$. Then, $Q: W \rightarrow W^{\prime}$ is a symplectomorphism and therefore $X$ is a symplectic manifold. In dimension $n=3$, it is not hard to see that $f$ coincides topologically with the example given in Construction 4.6 .

Remark 4.18. Observe that the symplectic structure in the example in Proposition 4.17 can be deformed by considering the 1 -form $\eta^{\prime}=\eta+d H$ for any $C^{\infty}$ function $H$. It turns out that there are cases for which $H$ does not induce a trivial deformation of the symplectic structure. In fact, the example in Proposition 4.17 belongs to a large family of Lagrangian fibrations whose members coincide topologically but may not be symplectomorphic.

Theorem 4.19. Let $\mathcal{F}=(X, \omega, f) \in \mathcal{L}(1,2)$. Then there are local sections $e_{1}, e_{2}, e_{3}$ of $R_{c}^{2} f_{*}^{\#} \mathbb{Z}$ such that the corresponding period 1 -forms are:

$$
\tau_{1}=\tau_{0}+d H, \quad \tau_{2}=2 \pi d b_{2}, \quad \tau_{3}=2 \pi d b_{3}
$$

where $\tau_{0}=\sum \alpha_{i} d b_{i}$ is as in Proposition 4.17, $\alpha_{1}$ is as in (22) and $H$ is a smooth function. Let $B \subseteq \mathbb{R}^{3}$ be an open ball. Secondly, for each $H \in$ $C^{\infty}(B)$ there is a fibration $\mathcal{F}_{H} \in \mathcal{L}(1,2)$ with periods $\tau_{1}, \tau_{2}, \tau_{3}$ as above. The monodromy representation of $\mathcal{F} \in \mathcal{L}(1,2)$ is generated by the matrices: $\left(\begin{array}{lll}1 & 0 & 0 \\ 1 & 1 & 0 \\ 0 & 0 & 1\end{array}\right),\left(\begin{array}{lll}1 & 0 & 0 \\ 0 & 1 & 0 \\ 1 & 0 & 1\end{array}\right),\left(\begin{array}{lll}1 & 0 & 0 \\ 1 & 1 & 0 \\ 1 & 0 & 1\end{array}\right)$.

Proof. The second statement follows from Proposition 4.17 and Remark 4.18. For the first claim, recall from Corollary 4.9 that any $\mathcal{F} \in \mathcal{L}(1,2)$ can be normalised in a neighbourhood $U \subset X$ of $p \in f^{-1}(0) \cap \operatorname{Crit}(f)$ by $F: U \cong \mathbb{C}^{3} \rightarrow B \subset \mathbb{R}^{3}, F=\left(F_{1}, F_{2}, F_{3}\right)$ as in (11). By redefining $X:=f^{-1}(f(U))$ if necessary, the restriction $\left.f\right|_{X \backslash U}$ induces a trivial bundle over $B$ with fibre $T^{2} \times[0,1]$. We can define sections $e_{1}, e_{2}, e_{3} \in R_{c}^{2} f_{*}^{\#} \mathbb{Z}$ in terms of the action of the Hamiltonian vector fields $v_{i}=v_{F_{i}}$ on $U$ and their extension to $X \backslash U$. For $i=2,3$ and $b \in B$ we take $e_{i}(b)$ represented by integral curves $\gamma_{i}:[0,2 \pi] \rightarrow F^{-1}(b)$ of $v_{i}$. For $e_{1}(b), b \in B \backslash \Delta$, we consider the sections $\Sigma_{1}:=\Sigma^{+}$and $\Sigma_{2}:=\Sigma^{-}$of $F$ as in Corollary 4.11 and define a representative $\gamma_{1}(b)$ of $e_{1}(b)$ as a suitable composition on flows of $v_{1}, v_{2}, v_{3}$, starting on $\Sigma_{1}(b)$ passing through $\Sigma_{2}(b)$ and returning to $\Sigma_{1}(b)$ in a completely analogous way as we did in the proof of Proposition 3.10. The reader may easily check that the period 1-forms computed over $\gamma_{i}$ are $\tau_{i}$ as claimed. 
It is well known that the monodromy about the singular fibre of a focusfocus fibration can be explained in terms of a Dehn twist. Similarly, for a fibration $\mathcal{F} \in \mathcal{L}(1,2)$, the monodromy of $\mathcal{F}$ can be understood as a "two dimensional Dehn twist". For each generator of $\pi_{1}(B \backslash \Delta, b)$, this twist is given by a full turn of a $T^{2}$-orbit, $\mathcal{T}(b)$, in one of the following ways:

1) once in the direction of $v_{2}$;

2) once in the direction of $v_{3}$;

3 ) the turn in 1) followed by the turn in 2).
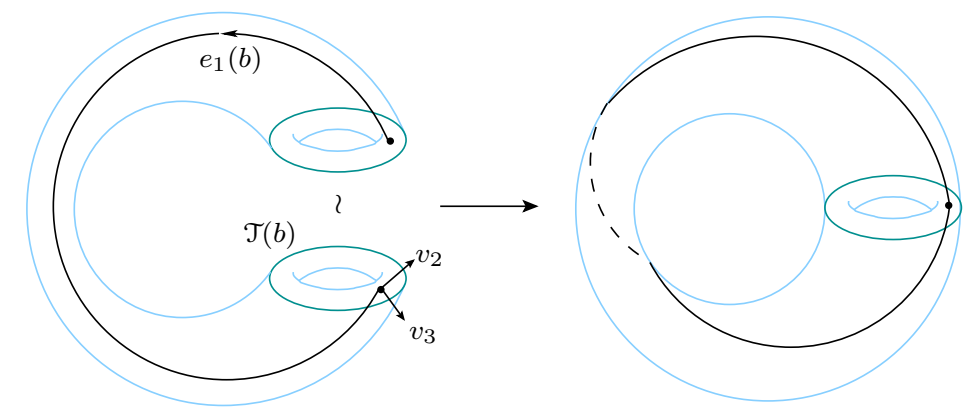

Figure 4. Monodromy around a component of $\Delta-\{0\}$.

In higher dimensions the description is analogous.

\section{The classification}

Let $\mathcal{F}=(X, \omega, f)$ be a Lagrangian $T^{3}$ fibration over a smooth manifold $B$ and let $\Delta \subset B$ be the discriminant locus of $f$. We are shall consider $\mathcal{F} \in \mathcal{L}(\kappa):=\mathcal{L}(\kappa, 2), \kappa=1,2$.

- Case $\kappa=1: B$ is an open 3-ball centred at $b_{0} \in \mathbb{R}^{3}, \Delta$ is a cone over 3 points. Let $b_{0} \in B$ be the vertex of $\Delta$. There is only one singular point $p$ on the fibre $X_{b_{0}}$, i.e., the Poisson orbit $O_{p}=\operatorname{Crit}\left(X_{b_{0}}\right)=p$. There is a neighbourhood $U \subset X$ of $p$ and a normal form $\left.\varphi \circ f\right|_{U}=q \circ \psi=F$ as in Corollary 4.9. The period lattice of $\mathcal{F}$ is as in Theorem 4.19.

- Case $\kappa=2: B=D \times(0,1), \Delta=\{0\} \times(0,1)$. Let $b_{0} \in \Delta$ and $X_{b_{0}}$ the fibre over $b_{0}$. A point $p \in \operatorname{Crit}(f)$ over $b_{0}$ belongs to a Poisson orbit $O_{p}=\operatorname{Crit}\left(X_{b_{0}}\right) \cong S^{1}$. There is a neighbourhood $U \subset X$ of $O_{p}$ and a normal form $\left.\varphi \circ f\right|_{U}=q \circ \psi=F$ as in Definition 3.6. The period lattice of $\mathcal{F}$ is as in Proposition 3.10.

Definition 5.1. Let $\left(b_{1}, b_{2}, b_{3}\right)$ be coordinates on $B$ and let $\phi \in C^{\infty}(B)$. Let $\partial_{J_{k}} \phi$ denote a partial derivative of $\phi$. We say that $\phi$ is $k$-flat at $\Delta$ if $\left.\partial_{J_{k}} \phi\right|_{b}=0$ for each $b \in \Delta$ and each $J_{k} \leq k$. If $k=\infty$ we say that $\phi$ is flat at $\Delta$. Let $\varphi: B \subseteq \mathbb{R}^{3} \rightarrow \mathbb{R}^{3}$ be a $C^{\infty}$ map, written as $\varphi=\left(\varphi_{1}, \varphi_{2}, \varphi_{3}\right)$. We 
say that $\varphi$ is tangent to the identity at $\Delta$ if for each $i=1,2,3$, the function $\varphi_{i}(b)-b_{i}$ is flat at $\Delta$.

Definition 5.2. Let $\alpha$ be a function on $B$ which is $C^{\infty}$ on $B \backslash \Delta$. We say that $\alpha$ is of rational type if for each $J_{k} \in \mathbb{Z}_{\geq 0}$ and any flat function $\phi$,

$$
\lim _{b \rightarrow \Delta} \phi \partial_{J_{k}} \alpha=0 \text {. }
$$

Example 5.3. The function $\alpha(s, r)=\log |s|$ on $D \times(0,1)$ is of rational type. Similarly, $\alpha$ as in Proposition 4.14 is also of rational type.

Definition 5.4. Let $\mathcal{F}, \mathcal{F}^{\prime} \in \mathcal{L}(\kappa), \kappa=1,2$, and let $\tau=\tau_{0}+d H$ and $\tau^{\prime}=\tau_{0}+d H^{\prime}$ be the singular periods of $\mathcal{F}$ and $\mathcal{F}^{\prime}$ respectively. We say that $\mathcal{F}$ is formally equivalent to $\mathcal{F}^{\prime}$ if the function $H-H^{\prime}$ is flat at $\Delta$.

Proposition 5.5. Let $\mathcal{F}=(X, \omega, f)$ and $\mathcal{F}^{\prime}=\left(X^{\prime}, \omega^{\prime}, f^{\prime}\right)$ in $\mathcal{L}(\kappa)$. Let $\tau_{i}$ and $\tau_{i}^{\prime}, i=1,2,3$, be the corresponding period 1 -forms. If $\mathcal{F}$ and $\mathcal{F}^{\prime}$ are formally equivalent there is a $C^{\infty}$-diffeomorphism between two small neighbourhoods of $b_{0} \in \Delta, \varphi: B \rightarrow \varphi(B)=: B^{\prime}$, such that $\varphi^{*}\left(\tau_{i}^{\prime}\right)=\tau_{i}$ for $i=1,2,3$. Conversely, if there is a diffeomorphism $\varphi$ of $B$ matching $\tau_{i}$ and $\tau_{i}^{\prime}$ and such that $\varphi$ is tangent to the identity, then $\mathcal{F}$ is formally equivalent to $\left(X^{\prime}, \omega^{\prime}, \varphi^{-1} \circ f^{\prime}\right)$.

Proof. Let $\tau:=\tau_{1}$ and $\tau^{\prime}:=\tau_{1}^{\prime}$ be the singular periods of $\mathcal{F}$ and $\mathcal{F}^{\prime}$, expressed in the coordinates $\left(b_{1}, b_{2}, b_{3}\right)$ as in the previous section. We want to find a diffeomorphism $\varphi$ such that $\varphi^{*} \tau^{\prime}=\tau$ and $\tau_{j}=\varphi^{*} \tau_{j}^{\prime}, j=2,3$. The latter implies that $\varphi$ should be of the form:

$$
b \mapsto\left(\varphi_{1}(b), b_{2}, b_{3}\right),
$$

where $\varphi_{1}$ is a smooth function to be determined. Now, for $t \in[0,1]$, we define a family of closed 1-forms $\tau_{t}=\tau+t\left(\tau^{\prime}-\tau\right)$. Then, $\left.\tau_{t}\right|_{t=0}=\tau$ and $\left.\tau_{t}\right|_{t=1}=$ $\tau^{\prime}$. Suppose there is a 1-parameter family of maps, $G_{t}$, varying smoothly with respect to $t$, such that each $G_{t}$ is a diffeomorphism between small neighbourhoods of $0 \in \mathbb{C}$ and such that $G_{0}$ is the identity map. Additionally, suppose that:

$$
\frac{d G_{t}^{*} \tau_{t}}{d t}=0
$$

Then, $G_{1}^{*} \tau_{t_{1}}=G_{0}^{*} \tau_{t_{0}}$ and we could define $\varphi:=G_{1}$. It is standard to realise $G_{t}$ by means of integrating a time dependent vector field $V_{t}$. Using Cartan identity, we can rewrite equation (30) as:

$$
G_{t}^{*}\left(\mathcal{L}_{V_{t}} \tau_{t}+\frac{d \tau_{t}}{d t}\right)=G_{t}^{*}\left(d\left(\iota_{V_{t}} \tau_{t}\right)+\tau^{\prime}-\tau\right)=0 .
$$

Observe that $\tau^{\prime}-\tau=d\left(H^{\prime}-H\right)$. Then, the solution to (31) is determined by

$$
\iota_{V_{t}} \tau_{t}=H-H^{\prime}
$$


The solution should be of the form $V_{t}=g_{t}(b) \partial_{b_{1}}$ where $g_{t}(b)$ is a smooth function of $b$ and $t$. We observe that, since we want $\varphi(\Delta)=\Delta$, then $V_{t}$ should satisfy $V_{t}(\Delta)=0$. The left hand side of equation (32) is:

$$
g_{t}(b) \cdot\left(\alpha(b)+\frac{\partial}{\partial b_{1}}\left(H+t\left(H^{\prime}-H\right)\right)\right) .
$$

Define

$$
g_{t}(b)=\frac{H-H^{\prime}}{\alpha(b)+\psi(b, t)}
$$

where $\psi(b, t)=\frac{\partial H}{\partial b_{1}}+t \frac{\partial\left(H^{\prime}-H\right)}{\partial b_{1}}$ is a $C^{\infty}$ function on $B \times[0,1]$. For $\kappa=1$ we know from Remark 4.16 that $\alpha$ blows up at $\Delta$ with order at most -1 . In the other hand, for $\kappa=2 \alpha$ blows up at $\Delta$ as a logarithm. Since $H-H^{\prime}$ vanishes at $\Delta$ to all orders, for $\kappa=1,2$ we have

$$
\lim _{b \rightarrow \Delta} \frac{\left(H-H^{\prime}\right)}{\alpha}=0 .
$$

Therefore $g_{t}$ is continuous and $g_{t}(b)=0$ when $b \in \Delta$. In particular $V_{t}(\Delta)=0$ as required. A similar argument can be used to prove the smoothness of $g_{t}$. Indeed, for $\kappa=1$ the estimates carried out in Proposition 4.15 show that all the functions $\partial_{J_{k}} \alpha$ blow up with finite order along $\Delta$. This implies that for any $h \in C^{\infty}(B)$ which is flat on $\Delta$,

$$
\lim _{b \rightarrow \Delta} h \partial_{J_{k}} \alpha=0 .
$$

Now observe that the $k$-th partial derivatives of $\left(H-H^{\prime}\right) / \alpha$ are finite sums of terms of the type

$$
\frac{h \partial_{J_{k}} \alpha}{\alpha^{m}}
$$

with $m \leq 2 k$ and $h \in C^{\infty}(B)$ flat at $\Delta$. It is not difficult to see from this that $\partial_{J_{k}} g_{t}$ is a continuous function on $B$ which vanishes at $\Delta$. A completely analogous argument is valid for the case $\kappa=2$. Therefore $g_{t}(s)$ is a $C^{\infty}$ function on $B$ and it is flat at $\Delta$. This implies that the time one map of $V_{t}(b), \varphi:=G_{1}$, is a diffeomorphism which is tangent to the identity on $\Delta$.

Now suppose there is a diffeomorphism $\varphi$ matching $\tau_{i}$ and $\tau_{i}^{\prime}$ which is tangent to the identity at $\Delta$. Since $\varphi^{*} \tau_{1}^{\prime}=\tau_{1}$ we can write $\varphi^{*} \tau_{0}-\tau_{0}=$ $d\left(H-H^{\prime} \circ \varphi\right)$. Furthermore, $\varphi$ can be written in coordinates $\left(b_{1}, b_{2}, b_{3}\right)$ as before as $\varphi=\left(\varphi_{1}, b_{2}, b_{3}\right)$, with $\varphi_{1}=\varphi_{1}(b)$ a smooth function on $B$. Observe that the 1-form $T:=\varphi^{*} \tau_{0}-\tau_{0}$ is single valued and smooth on $B$. Let us write $T=\sum T_{i} d b_{i}$. We claim that the functions $T_{i}$ are flat on $\Delta$. From this it follows directly that $H-H^{\prime} \circ \varphi$ is flat at $\Delta$. Now we will see that $\left.\partial_{J_{k}} T_{i}\right|_{\Delta}=0$ for all $i=1,2,3$ and for each $J_{k} \leq k, k \in \mathbb{Z}_{\geq 0}$. Recall that $\tau_{0}=\sum \alpha_{j} d b_{j}$, where $\alpha_{1}=\alpha$ is a function on $B$ of rational type (cf. 
Definition 5.2) and $\alpha_{2}$ and $\alpha_{3}$ are locally defined. After an easy calculation we obtain

$$
\begin{aligned}
& T_{1}=\left(\alpha_{1} \circ \varphi\right) \partial_{b_{1}} \varphi_{1}-\alpha_{1} \\
& T_{2}=\left(\alpha_{1} \circ \varphi\right) \partial_{b_{2}} \varphi_{1}+\alpha_{2} \circ \varphi-\alpha_{2} \\
& T_{3}=\left(\alpha_{1} \circ \varphi\right) \partial_{b_{3}} \varphi_{1}+\alpha_{3} \circ \varphi-\alpha_{3} .
\end{aligned}
$$

Since $\varphi$ is tangent to the identity at $\Delta,\left.\partial_{b_{1}} \varphi_{1}\right|_{\Delta}=1$ and $\partial_{b_{1}} \varphi_{1}(b)>0$ for $b$ in a small enough neighbourhood of $\Delta$. Then $T_{1}(b) \rightarrow 0$ as $b \rightarrow \Delta$ but since $T_{1}$ is continuous we have $\left.T_{1}\right|_{\Delta}=0$. Similarly, $\partial_{b_{2}} \varphi_{1}, \partial_{b_{3}} \varphi_{1}$ and all the higher order derivatives of $\varphi_{1}$ vanish when restricted to $\Delta$. In particular $\partial_{b_{2}} \varphi_{1}, \partial_{b_{3}} \varphi_{1}$ are flat on $\Delta$. Then $\left.\left(\alpha_{1} \circ \varphi\right) \partial_{b_{j}} \varphi_{1}\right|_{\Delta}=0$ for $j=2,3$. Now let us consider a (perhaps smaller) neighbourhood of $\Delta$ and a branch of $\alpha_{j}$ in this neighbourhood. Since $\varphi$ is infinitely tangent to the identity at $\Delta$, then $\left(\alpha_{j} \circ \varphi-\alpha_{j}\right)(b) \rightarrow 0$ as $b \rightarrow \Delta$. From the continuity of $T_{j}$ we have $\left.T_{j}\right|_{\Delta}=0$ for $j=2,3$. One can use the argument above inductively to show that $\partial_{J_{k}} T_{i}$ vanish on $\Delta$ for $k \geq 1$.

Proposition 5.6. Let $\mathcal{F}$ and $\mathcal{F}^{\prime}$ be in $\mathcal{L}(\kappa)$. Let $\varphi: B \rightarrow \varphi(B)=: B^{\prime}$ be a diffeomorphism, such that $\varphi(\Delta)=\Delta$ and $\varphi^{*}\left(\tau_{i}^{\prime}\right)=\tau_{i}$ for $i=1,2,3$. Then, there are sections $\Sigma$ and $\Sigma^{\prime}$ of $f$ and $f^{\prime}$ and a commutative diagram:

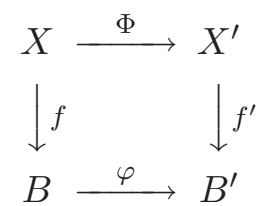

where $\Phi$ is an orientation preserving diffeomorphism sending $\Sigma$ to $\Sigma^{\prime}$. The map $\Phi$ can be assumed to be equivariant with respect to the $T^{2}$-actions induced by $\tau_{j}$ and $\tau_{j}^{\prime} j=2,3$. Furthermore, if $\Sigma$ and $\Sigma^{\prime}$ are Lagrangian, then $\Phi$ is a symplectomorphism.

Proof. Recall that $\mathcal{F}$ is normalised in a neighbourhood $U \subset X$ of the critical Poisson orbit $O_{p} \subset X_{b_{0}}$ by means of a symplectomorphism $\psi: U \rightarrow\left(V, \omega_{0}\right)$. Similarly, for $\mathcal{F}^{\prime}$ there is a neighbourhood $U^{\prime} \subset X^{\prime}$ of $O_{p^{\prime}}$ and a symplectomorphism $\psi^{\prime}: U^{\prime} \rightarrow\left(V, \omega_{0}\right)$.

Let $W \subseteq \psi(U) \cap \psi^{\prime}\left(U^{\prime}\right)$. For simplicity denote $U:=\psi^{-1}(W)$ and define $\Phi_{0}:=\left.\left(\psi^{\prime}\right)^{-1} \circ \psi\right|_{U}$. Then, $\Phi_{0}$ is a symplectomorphism such that $\Phi_{0}\left(O_{p}\right)=$ $O_{p^{\prime}}$ and such that $\varphi \circ F=F^{\prime} \circ \Phi_{0}$. Now let $\Sigma$ be a section of $F$ which does not pass through $O_{p}$. Defining $\Sigma^{\prime}=\Phi_{0}(\Sigma)$ gives a section of $F^{\prime}$ which does not pass through $O_{p^{\prime}}$. Notice that $\Sigma$ and $\Sigma^{\prime}$ also define sections of $f$ and $f^{\prime}$ respectively. Since $\Phi_{0}$ is a symplectomorphism, if $\Sigma$ is Lagrangian, then $\Sigma^{\prime}$ is Lagrangian too.

Let $\alpha$ be a local section of $T_{B}^{*}$. Let $v_{\alpha}$ be the vector field determined by the equation:

$$
F^{*} \alpha=\iota\left(v_{\alpha}\right) \omega
$$


If we consider the 1 -form, $d b_{i}$, then $v_{d b_{i}}=v_{q_{i}}$. As we observed before, each $v_{q_{i}}$ extends to a vector field on $X$ which is tangent to the fibres of $f$. Therefore, $v_{\alpha}$ extends to $X$ and, since the fibres of $f$ are compact, the flow $g_{\alpha}^{t}$ of $v_{\alpha}$ is defined for all $t \in \mathbb{R}$. For each $\alpha$ define the map $T_{\alpha}:=g_{\alpha}^{1}: X \rightarrow X$. It follows that $\alpha \mapsto T_{\alpha}$ induces a fibre preserving action, $T: T_{B}^{*} \times_{B} X \rightarrow X$. Now define the map $\tilde{\pi}: T_{B}^{*} \rightarrow X$ such that for each $\alpha_{b} \in T_{B, b}^{*}, \tilde{\pi}\left(\alpha_{b}\right)=T_{\alpha}(\Sigma(b))=: x$, which lies on the fibre $f^{-1}(b)$. One can verify that $x$ only depends on the value of $\alpha$ at $b$. So, for $\bar{\alpha}$ such that $\bar{\alpha}(b)=\alpha(b), T_{\bar{\alpha}}(\Sigma(b))=T_{\alpha}(\Sigma(b))=x$.

Let $Z$ be the zero section on $T_{B}^{*}$. We know from Theorem 2.5 that $\tilde{\pi}\left(T_{B}^{*}\right)=$ $X^{\#}, \tilde{\pi}^{-1}(\tilde{\pi}(Z))=\Lambda$ is the period lattice of $f$ and $\left.\tilde{\pi}^{-1}\right|_{X \#}: X^{\#} \rightarrow T_{B}^{*}$ is well defined modulo $\Lambda$. Moreover, $\left.\tilde{\pi}^{-1}\right|_{X^{\#}}$ composed with the projection $T_{B}^{*} \rightarrow T_{B}^{*} / \Lambda=J_{f}$ gives a diffeomorphism $X^{\#} \cong J_{f}$. If $\Sigma$ is Lagrangian this map is a symplectomorphism.

Now let us take $\alpha^{\prime}=\left(\varphi^{-1}\right)^{*} \alpha$; this is a local section of $T_{B^{\prime}}^{*}$. Consider the vector field $v_{\alpha^{\prime}}$ induced by $\left(F^{\prime}\right)^{*} \alpha^{\prime}=\iota\left(v_{\alpha^{\prime}}\right) \omega^{\prime}$. Let $g_{\alpha^{\prime}}^{t}$ be the flow of $v_{\alpha^{\prime}}$. Again, this flow is complete, so we can define $T_{\alpha^{\prime}}: X^{\prime} \rightarrow X^{\prime}$ such that $T_{\alpha^{\prime}}:=g_{\alpha^{\prime}}^{1}$. Let $\tilde{\pi}^{\prime}: T_{B^{\prime}}^{*} \rightarrow X^{\prime}$ such that $\tilde{\pi}^{\prime}\left(\alpha_{b^{\prime}}^{\prime}\right)=T_{\alpha^{\prime}}\left(\Sigma^{\prime}\left(b^{\prime}\right)\right)=: x^{\prime}$. Define the map $\Phi^{\#}: X^{\#} \rightarrow X^{\prime \#}$ as the composition:

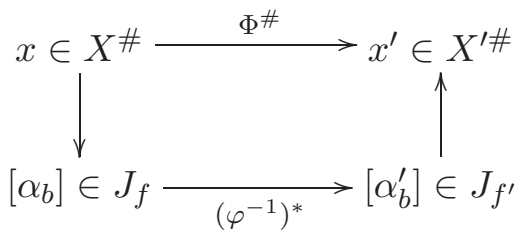

The horizontal map, which is induced by the pull back of sections under $\varphi$, is well defined as $\varphi^{*}$ sends $\Lambda^{\prime}$ to $\Lambda$. The vertical maps, e.g. $\left[\alpha_{b}\right] \mapsto g_{\alpha}^{1}(\Sigma(b))$, are independent of the choice of the representative of $\left[\alpha_{b}\right] \in J_{f}$. Indeed, let $\tilde{\alpha}_{b} \in\left[\alpha_{b}\right]$. Then, $\tilde{\alpha}_{b}=\alpha_{b}+\lambda_{b}$, with $\lambda_{b} \in \Lambda_{b}$. It follows that $g_{\alpha+\lambda}^{t}=g_{\alpha}^{t}$. In particular, $g_{\alpha+\lambda}^{1}(\Sigma(b))=g_{\alpha}^{1}(\Sigma(b))$.

We can write explicitly,

$$
\Phi^{\#}(x)=g_{\alpha^{\prime}}^{1}\left(\Sigma^{\prime}\left(b^{\prime}\right)\right)
$$

where $x=g_{\alpha}^{1}(\Sigma(b))$ for some $\left[\alpha_{b}\right] \in J_{f}$ and $\alpha^{\prime}=\left(\varphi^{-1}\right)^{*} \alpha$. Notice that $\varphi$ induces a symplectomorphism between $T_{B^{\prime}}^{*}$ and $T_{B}^{*}$. Hence $\Phi^{\#}$ is a diffeomorphism and, when $\Sigma$ is Lagrangian, $\Phi^{\#}$ is a symplectomorphism.

Now let $X^{\#} \hookrightarrow X$ be the inclusion map and consider $x \in U \cap X^{\#}$ over $b \in B$. We define

$$
\Phi(x)= \begin{cases}\Phi^{\#}(x), & x \in X^{\#} \\ \Phi_{0}(x) & x \in U .\end{cases}
$$


The map $\Phi$ extends $\Phi^{\#}$ to $X$ and the $T^{2}$-equivariance of $\Phi$ is verified $a$ priori. $\Phi$ is $C^{\infty}$ since the map $J_{f} \rightarrow J_{f^{\prime}}$ is. We still need to check, however, that $\Phi^{\#}(x)=\Phi_{0}(x)$ on $U \cap X^{\#}$, i.e., that $\Phi$ is well defined. We prove this next.

Let $x \in U \cap X^{\#}$ over $b \in B$ and define $v_{\alpha}^{\prime}:=\Phi_{0 *}\left(v_{\alpha}\right)$ and let $g_{v_{\alpha}^{\prime}}^{t}$ denote the flow of $v_{\alpha}^{\prime}$. We claim that the equation $x=g_{\alpha}^{1}(\Sigma(b))$ implies that

$$
\Phi_{0}(x)=g_{v_{\alpha}^{\prime}}^{1}\left(\Phi_{0}(\Sigma(b))\right)
$$

To see this let us regard $\gamma(t):=g_{\alpha}^{t}(\Sigma(b))$ as the integral curve of $v_{\alpha}$ such that $\gamma(0)=\Sigma(b)$ and $\gamma(1)=x$. Now let $\gamma^{\prime}(t):=\Phi_{0}(\gamma(t))$. This is a curve on $F^{\prime-1}\left(b^{\prime}\right), b^{\prime}=\varphi(b)$, such that $\gamma^{\prime}(0)=\Phi_{0}(\Sigma(b))=\Sigma^{\prime}\left(b^{\prime}\right)$ and $\gamma^{\prime}(1)=\Phi_{0}(x)$. Furthermore, $\gamma^{\prime}$ is an integral curve of $v_{\alpha}^{\prime}$. Indeed, we see that:

$$
\frac{d \gamma^{\prime}}{d t}=\frac{d\left(\Phi_{0} \circ \gamma\right)}{d t}=\Phi_{0 *}\left(v_{\alpha}\right)=v_{\alpha}^{\prime}
$$

Therefore $\gamma^{\prime}(t)=g_{v_{\alpha}^{\prime}}^{t}$ and $g_{v_{\alpha}^{\prime}}^{1}\left(\Sigma^{\prime}\left(b^{\prime}\right)\right)=\Phi_{0}(x)$. Now observe that $\Phi_{0}^{*} \omega^{\prime}=\omega$ implies that:

$$
v_{\alpha}^{\prime}=v_{\alpha^{\prime}}
$$

To prove this we notice that $F^{*} \alpha=\Phi_{0}^{*}\left(F^{\prime *} \alpha^{\prime}\right)$. Now we can write (35) as:

$$
\Phi_{0}^{-1 *}\left(\iota\left(v_{\alpha}\right) \Phi_{0}^{*} \omega^{\prime}\right)=F^{*} \alpha^{\prime}
$$

The left hand side of (40) can be written as $\iota\left(\Phi_{0 *} v_{\alpha}\right) \omega^{\prime}$. Then, it follows that $v_{\alpha}^{\prime}=\Phi_{0 *} v_{\alpha}=v_{\alpha^{\prime}}$. Now, from (38) and (39) we conclude that:

$$
\Phi_{0}(x)=g_{\alpha^{\prime}}^{1}\left(\Sigma^{\prime}\left(b^{\prime}\right)\right)
$$

which is equal to $\Phi^{\#}(x)$ in (37), hence $\Phi$ is well defined.

Observe that, for $\kappa=2$, we can start the above construction in terms of the section $\Sigma=\Sigma_{1}$ as in Construction 3.8, which is Lagrangian. Therefore $\Phi$ turns out to be a symplectomorphism. In the case $\kappa=1$, the sections $\Sigma^{ \pm}$as in (18) are not Lagrangian. This does not give much trouble as we can always find a Lagrangian section. The argument is valid for $\kappa=1,2$. Let $U \subset X$ be as before. Observe that for any given section $\Sigma_{0}$ of $f$ with $\Sigma_{0}(B) \subset U$, there exists a neighbourhood $\mathcal{U} \subseteq U \subset X$ of $\Sigma_{0}$ such that $\mathcal{U} \cap \operatorname{Crit}(f)=\varnothing$ and a fibre-preserving symplectomorphism $(\mathcal{U}, \omega \mid \mathcal{U}) \rightarrow$ $\left(T_{B}^{*}, \Omega\right)$. Here $\Omega=\omega_{0}+\beta$ where $\omega_{0}$ is the standard symplectic structure on $T_{B}^{*}$ and $\beta$ is the pull-back under $T_{B}^{*} \rightarrow B$ of a closed 2-form on $B$ (if $\Sigma_{0}$ were Lagrangian $\beta=0$ ). Observe that in our situation $\beta$ can be assumed to be exact, so we have $d \theta=\Omega-\omega_{0}$ for some 1-form $\theta$ on $B$. Then $-\theta$ defines a section, $\Sigma_{\theta}$, of $T_{B}^{*}$ which is Lagrangian with respect to $\Omega$. Then $\Sigma_{\theta}$ maps to a Lagrangian section, $\Sigma$, of $f$ inside $\mathcal{U}$. Using $\Sigma$ to define $\Phi$ we obtain a symplectomorphism. 
Theorem 5.7. Let $\mathcal{F}=(X, \omega, f)$ and $\mathcal{F}^{\prime}=\left(X^{\prime}, \omega^{\prime}, f^{\prime}\right)$ be Lagrangian fibrations of type $\mathcal{L}(\kappa), \kappa=1,2$. Then $\mathcal{F}$ is formally equivalent to $\mathcal{F}^{\prime}$ if and only if $\mathcal{F}$ is symplectically equivalent to $\mathcal{F}^{\prime}$.

Proof. Assume $\mathcal{F}$ and $\mathcal{F}^{\prime}$ are formally equivalent. Then Proposition 5.5 gives us a diffeomorphism $\varphi$ on $B$ such that $\varphi^{*} \tau_{j}^{\prime}=\tau_{j}$. In view of Proposition 5.6, $\varphi$ lifts to a fibre-preserving symplectomorphism $\Phi: X \rightarrow X^{\prime}$.

To prove the converse we suppose there is a symplectomorphism $\Psi$ and a suitable diffeomorphism $\varphi$, making a commutative diagram:

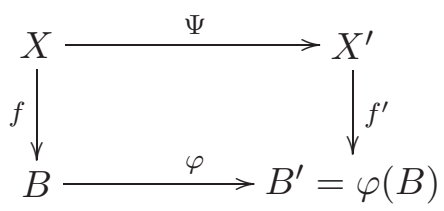

One can always take a diffeomorphism, $\tilde{\varphi}$, from a neighbourhood of $b_{0} \in B$ into a neighbourhood, $\tilde{B} \subseteq B^{\prime}$, of $\varphi\left(b_{0}\right)=b_{0}^{\prime}$ and such that $\varphi \circ \tilde{\varphi}^{-1}$ is tangent to the identity at $\Delta \cap \tilde{B}$. Let $\tilde{f}=\tilde{\varphi} \circ f$. Then, $(X, \omega, \tilde{f})$ and $\mathcal{F}$ define the same germ. Now, $\Psi$ together with the map $\varphi^{\prime}:=\varphi \circ \tilde{\varphi}^{-1}: \tilde{B} \rightarrow \varphi^{\prime}(\tilde{B}) \subseteq B^{\prime}$ makes $(X, \omega, \tilde{f})$ and $\mathcal{F}^{\prime}$ symplectomorphic, with $\varphi^{\prime}$ being tangent to the identity at $\Delta$. Let us denote $f:=\tilde{f}$ and $\varphi:=\varphi^{\prime}$.

We claim now that $\tau_{i}=\varphi^{*} \tau_{i}^{\prime}$. To see this we take $V_{i}^{\prime}$ to be the vector fields determined by the equation

$$
f^{\prime *} \tau_{i}^{\prime}=\iota_{V_{i}^{\prime}} \omega^{\prime}, \quad i=1,2,3 .
$$

These vector fields are defined on open sets $f^{\prime-1}\left(U^{\prime}\right)$, where $U^{\prime} \subset B_{0}^{\prime}$ is an open set on which a branch of $\tau_{1}^{\prime}$ is defined. It follows that $V_{i}^{\prime}$ are vector fields whose flows are periodic. We can take integral curves of $V_{i}^{\prime}$ to define simple loops, $\gamma_{i}^{\prime}(b)$ and on $f^{\prime-1}(b)$, representing the cycles $e_{i}^{\prime}(b)$ generating $H_{1}\left(f^{\prime-1}\left(b^{\prime}\right), \mathbb{R}\right)$. These loops can be used for computing the period 1-forms of $f^{\prime}$ which are, tautologically, $\tau_{i}^{\prime}$. Now define $V_{i}$ to be the vector fields determined by the equation $\iota\left(V_{i}\right) \omega=\left(f^{\prime} \circ \Psi\right)^{*} \tau_{i}$. Since $\Psi$ is symplectic, $\Psi_{*} V_{i}=V_{i}^{\prime}$. The above implies that the flow of $V_{i}$ is periodic. One verifies that suitable integral curves $\gamma_{i}$ of $V_{i}$ generate $H_{1}\left(X_{b}, \mathbb{Z}\right)$, so we can define the period one forms, $\tau_{i}$ of $f$ by integrating along $\gamma_{i}$. Now observe that, since the diagram (41) commutes, $V_{i}$ also satisfies the equation $\iota\left(V_{i}\right) \omega=\varphi^{*} \tau_{i}^{\prime}$. Therefore, $\tau_{i}=\varphi^{*} \tau_{i}^{\prime}$. The conclusion follows now from Proposition 5.5.

Acknowledgements. I would like to thank M. Gross, my thesis advisor, for his help and support. I am also grateful to D. Matessi, J. Rawnsley and R. Thomas for their useful comments and suggestions. I would like to thank the Mathematics Institute of Warwick University, U.K. and the I.C.T.P. in Trieste. I also wish to thank the referee of this article for making 
helpful suggestions that improved the final version of this paper, specially for pointing out to me a way to simplify $\S 5$.

\section{References}

[1] R. Castano-Bernard, Classification of Lagrangian Fibrations, Ph.D. thesis, Warwick University, 2002.

[2] J.J. Duistermaat, On global action-angle coordinates, Comm. Pure Appl. Math. 6 (1980), 678-706.

[3] L. Eliasson, Hamiltonian systems with Poisson commuting integrals, Ph.D. thesis, Stockholm, 1984.

[4] Ji-X. Fu, Rigidity of a class of special lagrangian fibrations singularity, Asian J. Math. 6(4) (2002), 663-678.

[5] M. Gross, Special Lagrangian Fibrations I: Topology, in 'Integrable systems and algebraic geometry (Kobe/Kyoto, 1997)', World Sci. Publishing, 156-193, River Edge, NJ, 1998, arXiv: alg-geom/9710006.

[6] _ Special Lagrangian Fibrations II: Geometry, Surv. Differ. Geom. 5 (1999), 341-403, arXiv: math.AG/9809072.

[7] _ Examples of special Lagrangian fibrations, in 'Symplectic geometry and mirror symmetry (Seoul, 2000),' 81-109, World Sci. Publishing, River Edge, NJ, 2001, arXiv: math.AG/0012002.

[8] _ Topological Mirror Symmetry, Invent. Math. 144 (2001), 75-137.

[9] M. Gross, D. Huybrechts, and D. Joyce, Calabi-Yau manifolds and related geometries (Lecture notes at a summer school in Nordfjordeid, Norway, June 2001), Springer Verlag, 2003.

[10] M. Gross and P.M.H. Wilson, Mirror symmetry via 3-tori for a class of Calabi-Yau threefolds, Math. Ann 309 (1997), 505-531.

[11] _ Large Complex Structure Limits of K3 Surfaces, J. Differential Geom. 55(3) (2000), 475-546.

[12] V. Guillemin and S. Sternberg, Convexity Properties of the Moment Mapping, Inv. Math. 67 (1982), 491-513.

[13] R. Harvey and H.B. Lawson, Jr., Calibrated Geometries, Acta Mathematica 148 (1982), 47-157.

[14] D. Joyce. Special Lagrangian $m$-folds in $\mathbb{C}^{m}$ with symmetries, Duke Math. J. 115(1) (2002), 1-51, arXiv: math.DG/0008021.

[15] _ Singularities of special Lagrangian fibrations and the $S Y Z$ conjecture, Communications in Analysis and Geometry 11(5) (2003), 859-907, arXiv: math.DG/ 0011179.

[16] M. Kontsevich and Y. Soibelman, Homological mirror symmetry and torus fibrations, in 'Symplectic geometry and mirror symmetry (Seoul, 2000),' 203-263, World Sci. Publishing, River Edge, NJ, 2001, arXiv: math.DG/0011041.

[17] E Miranda and N.-T. Zung, Equivariant normal form for non-degenerate singular orbits of integrable hamiltonian systems, preprint, 2003, arXiv: math.SG/0302287. 
[18] W.-D. Ruan, Lagrangian torus fibration of quintic hypersurfaces. I: Fermat quintic case, in 'Winter School on Mirror Symmetry, Vector Bundles and Lagrangian Submanifolds (Cambridge, MA, 1999)', AMS/IP Stud. Adv. Math., 23, 297-332, Amer. Math. Soc., Providence, RI, 2001.

[19] _ Lagrangian torus fibration of quintic Calabi-Yau hypersurfaces II: technical results on gradient flow construction, Journal of Symplectic Geometry 1 (2002), 435521.

[20] L L L Lagrangian torus fibration of quintic Calabi-Yau hypersurfaces III: symplectic topological SYZ mirror construction for general quintics, J. Differential Geometry 63 (2003), 171-229.

[21] E. Strominger, S.-T. Yau and E. Zaslow, Mirror symmetry is T-duality, Nucl. Phys. B 479 (1996), 243-259.

[22] San Vu-Ngoc, On semi-global invariants for focus-focus singularities, Topology 42(2) (2003), 365-380.

International Centre for Theoretical Physics

Mathematics SeCtion

Strada Costiera 11, Trieste 34014

ITALY

E-mail address: rcastano@ictp.trieste.it

Supported by the National Council for Science and Technology of Mexico (CONACYT) grant 116167 and the Abdus Salam International Centre for Theoretical Physics (ICTP), Trieste, Italy. 